\title{
The Plasma Wave Experiment (PWE) on board the Arase (ERG) satellite
}

\author{
Yoshiya Kasahara ${ }^{1 *}$,, Yasumasa Kasaba², Hirotsugu Kojima ${ }^{3}$, Satoshi Yagitani ${ }^{1}$, Keigo Ishisaka4, \\ Atsushi Kumamoto ${ }^{2}$, Fuminori Tsuchiya ${ }^{2}$, Mitsunori Ozaki ${ }^{1}$, Shoya Matsuda ${ }^{5}$, Tomohiko Imachi ${ }^{1}$, \\ Yoshizumi Miyoshi ${ }^{5}$, Mitsuru Hikishima ${ }^{6}$, Yuto Katoh ${ }^{2}$, Mamoru Ota $^{1}$, Masafumi Shoji ${ }^{5}$, Ayako Matsuoka ${ }^{6}$ \\ and Iku Shinohara ${ }^{6}$
}

\begin{abstract}
The Exploration of energization and Radiation in Geospace (ERG) project aims to study acceleration and loss mechanisms of relativistic electrons around the Earth. The Arase (ERG) satellite was launched on December 20, 2016, to explore in the heart of the Earth's radiation belt. In the present paper, we introduce the specifications of the Plasma Wave Experiment (PWE) on board the Arase satellite. In the inner magnetosphere, plasma waves, such as the whistlermode chorus, electromagnetic ion cyclotron wave, and magnetosonic wave, are expected to interact with particles over a wide energy range and contribute to high-energy particle loss and/or acceleration processes. Thermal plasma density is another key parameter because it controls the dispersion relation of plasma waves, which affects wave-particle interaction conditions and wave propagation characteristics. The DC electric field also plays an important role in controlling the global dynamics of the inner magnetosphere. The PWE, which consists of an orthogonal electric field sensor (WPT; wire probe antenna), a triaxial magnetic sensor (MSC; magnetic search coil), and receivers named electric field detector (EFD), waveform capture and onboard frequency analyzer (WFC/OFA), and high-frequency analyzer (HFA), was developed to measure the DC electric field and plasma waves in the inner magnetosphere. Using these sensors and receivers, the PWE covers a wide frequency range from DC to $10 \mathrm{MHz}$ for electric fields and from a few $\mathrm{Hz}$ to $100 \mathrm{kHz}$ for magnetic fields. We produce continuous ELFNLF/HF range wave spectra and ELF range waveforms for $24 \mathrm{~h}$ each day. We also produce spectral matrices as continuous data for wave direction finding. In addition, we intermittently produce two types of waveform burst data, "chorus burst" and "EMIC burst." We also input raw waveform data into the software-type wave-particle interaction analyzer (S-WPIA), which derives direct correlation between waves and particles. Finally, we introduce our PWE observation strategy and provide some initial results.
\end{abstract}

Keywords: Plasma wave, Radiation belt, Geospace, Inner magnetosphere, Chorus

\section{Introduction}

In the Earth's magnetosphere, various plasma phenomena simultaneously occur at various time, spatial, and energy scales. The generation and disappearance mechanisms of the Earth's radiation belt are critical issues in space weather and the science of magnetized planets, such as Jupiter and Saturn. It is well known that the high-energy particle population trapped in the Earth's radiation belt

\footnotetext{
*Correspondence: kasahara@is.t.kanazawa-u.ac.jp

${ }^{1}$ Graduate School of Natural Science and Technology, Kanazawa

University, Kakuma, Kanazawa 920-1192, Japan

Full list of author information is available at the end of the article
}

significantly changes during the magnetically disturbed period of magnetic storms and high-speed solar wind streams (HSS) (e.g., Paulikas and Blake 1979; Reeves et al. 2003). Since Summers et al. (1998) proposed that relativistic electrons in the outer belt are produced by acceleration with the whistler-mode chorus, many studies have investigated the relationship between outer radiation belt variation and the whistler-mode chorus during a magnetic storm "recovery" (e.g., Miyoshi et al. 2003; Horne et al. 2005; Kasahara et al. 2009; Thorne 2010; Turner et al. 2013). Kasahara et al. (2004) used statistical methods to study the spatial distribution of the whistler-mode chorus during magnetic storm recovery phases with data 
from the VLF instruments (Kimura et al. 1990) on board the Akebono satellite. This study clarified that the whistler-mode chorus is primarily located near the inner edge of the outer radiation belt $(L \sim 2.5)$ and gradually shifts outward $(L \sim 4)$ with time and that the total wave energy generated during the storm is large enough to accelerate relativistic electrons. Recently, Miyoshi et al. (2013) demonstrated that whistler-mode waves accelerate relativistic electrons during HSS events with southward interplanetary magnetic field (IMF)-dominant HSS (SBz-HSS), while this acceleration mechanism is not effective during HSS events with northward IMF-dominant HSS (NBzHSS). They finally suggested that the responses of the outer radiation belt flux depend on the whistler-mode wave-electron interactions that are associated with a series of sub-storms.

It is well established that there are various types of plasma waves other than the whistler-mode chorus in the equatorial region, such as electromagnetic ion cyclotron wave (EMIC) (Young et al. 1981; Kasahara et al. 1992; Sakaguchi et al. 2013; Min et al. 2012; Usanova et al. 2012; Matsuda et al. 2014a; and the references therein) and magnetosonic wave (MSW) (Laakso et al. 1990; Kasahara et al. 1994; Fraser and Nguyen 2001; Santolik et al. 2004, 2016; and the references therein). It was also suggested that in addition to the whistler-mode chorus, the MSW plays an important role in acceleration processes in the inner magnetosphere (e.g., Horne et al. 2007; Meredith et al. 2008). On the other hand, pitch angle scattering caused by the EMIC is thought to be one plausible loss mechanism that contributes to relativistic electron precipitation (Miyoshi et al. 2008; Jordanova et al. 2008; Shprits et al. 2016). Furthermore, there are additional suggestions that plasmaspheric hiss (Breneman et al. 2015) and VLF radio transmitters (Baker et al. 2014) could play important roles in the electron loss process in the radiation belt.

As described above, plasma waves from several $\mathrm{Hz}$ to a few tens of $\mathrm{kHz}$, such as the whistler-mode chorus, EMIC, and MSW, are expected to interact with charged particles over a wide energy range from a few $\mathrm{eV}$ to $\mathrm{MeV}$ and contribute to high-energy particle loss and/or acceleration processes in the inner magnetosphere. In these processes, thermal plasma density is a key parameter because it controls plasma wave dispersion relations, which affect wave-particle interaction conditions and wave propagation characteristics. In addition, DC electric field also plays an important role in controlling the global dynamics of the inner magnetosphere (e.g., Nishimura et al. 2007; Thaller et al. 2015; and the references therein).

Computer simulation is a powerful tool that can be used to understand the complex physical processes in the inner magnetosphere. Recent computer simulations have clarified that the chorus emission is effectively generated in the region close to the magnetic equator through nonlinear wave-particle interactions (e.g., Katoh and Omura 2007; Omura et al. 2008). Omura (2014) reviewed a nonlinear theory of the generation mechanism of chorus emission, describing the nonlinear dynamics of resonant electrons and the electromagnetic electron "hole" formation in phase space that results in resonant currents generating rising-tone emissions. Conversely, fallingtone emissions are generated through the formation of electron "hills" (Omura 2014). Katoh and Omura (2016) carried out a self-consistent simulation of the whistlermode chorus generation process and showed that simulated spectral fine structures were similar to the Cluster spacecraft observation. They also suggested that oblique propagation of waves with respect to the magnetic field is essential for forming the half gyrofrequency gap. Omura et al. (2015) demonstrated that chorus emissions can accelerate electrons from tens of $\mathrm{keV}$ to several $\mathrm{MeV}$ within a few minutes and that nonlinear trapping processes [i.e., relativistic turning acceleration (RTA) and ultra-relativistic acceleration (URA)] form a dumbbell distribution of relativistic electrons.

Wave-particle interactions related to EMIC waves have also been vigorously studied. Shoji and Omura (2014) performed parametric analyses of EMIC-triggered emissions with a gradient of non-uniform ambient magnetic field using a hybrid simulation. Nakamura et al. (2015) investigated sub-packet structures found in EMIC risingtone emissions observed by THEMIS, showing that the time evolution of the observed frequency and amplitude can be reproduced consistently by nonlinear growth theory. Kubota et al. (2015) performed test particle simulations of relativistic electrons interacting with EMICtriggered emissions in the plasmasphere and concluded that EMIC-triggered emissions can efficiently precipitate electrons at $0.5-6 \mathrm{MeV}$.

The Exploration of energization and Radiation in Geospace (ERG) project aims to study relativistic electron acceleration and loss mechanisms around the Earth (Miyoshi et al. 2012, 2017). The ERG satellite, launched on December 20, 2016, and nicknamed "Arase," is a satellite to explore the Earth's radiation belt using electric and magnetic field instruments and electron and ion detectors covering wide frequency and energy ranges, respectively. The inclination of the Arase is $31^{\circ}$, and the altitudes of the apogee and perigee are $\sim 32,000$ and $460 \mathrm{~km}$, respectively. The orbital period is approximately $570 \mathrm{~min}$. The Arase satellite is a spin-stabilized satellite, with its spin axis pointed $15^{\circ}$ toward the sun (within $4^{\circ}-20^{\circ}$ ) and a spin period of $\sim 8 \mathrm{~s}$.

The ERG project includes ground-network observations and integrated studies/simulation teams (Miyoshi 
et al. 2012, 2017). Conjugate observations of the Arase and the ground observation network are important in comprehensive understanding of particle precipitation processes, as consequences of wave-particle interactions. Furthermore, collaborative studies between observation and simulation are essential to clarify the multiscale plasma dynamics, in which macroscopic and microscopic processes complicatedly occur interacting with each other, in the inner magnetosphere. In these contexts, measurements of plasma waves are required to cover wide frequency ranges in both electric and magnetic fields to observe various plasma waves interacting with ions and electrons over wide energy ranges. In particular, it is very important to capture continuous waveform as long as possible with a precise time resolution to analyze the phase difference between wave packets and particles, and also the time difference between measurements by the Arase and ground-network stations.

The NASA Van Allen Probes, formerly called Radiation Belt Storm Probes (RBSP), began their observations of the inner magnetosphere in 2012 (Mauk et al. 2013), prior to the launch of the Arase satellite. As for the measurements of the DC electric field and plasma waves, the EFW (Wygant et al. 2013) and the EMFISIS (Kletzing et al. 2013) on board the Van Allen Probes have resulted in many scientific achievements over the past 5 years. Although the orbit of the Arase satellite is quite similar to the orbits of the Van Allen Probes, the inclination of Arase $\left(31^{\circ}\right)$ is much larger than the ones of Van Allen Probes $\left(\sim 10^{\circ}\right)$, which will allow for the ability to explore not only the equatorial region but also the off-equatorial region. We expect to measure plasma waves such as chorus and EMIC propagating from their generation regions near the equatorial plane and derive their plasma wave properties (e.g., wave normal direction and Poynting flux) that could be also important key parameters to clarify the wave-particle interaction along their propagation paths in the off-equatorial region.

Figure 1 shows an overview of the Arase satellite. The "SGA" subscript in the coordinate system stands for "Spinning Satellite Geometry Axis" coordinates, which represent the geometrical positions of the components on board the satellite. The Arase has two sets of MAST named MAST-MGF and MAST-SC. A triaxial fluxgate magnetometer (referred to as MGF) is mounted on the top of the MAST-MGF to measure magnetic fields from DC to a few tens of $\mathrm{Hz}$ (Matsuoka et al. 2018). On the top of the MAST-SC, a triaxial magnetic search coil (referred to as MSC) is mounted to measure AC magnetic fields from a few $\mathrm{Hz}$ to $100 \mathrm{kHz}$ (Ozaki et al. 2018). The WPT consists of four wire antennas (wire probe antenna sensor; WPT-S) to measure electric fields from DC to $10 \mathrm{MHz}$ (Kasaba et al. 2017). The branch numbers attached after WPT-S (i.e., WPT-S1, WPT-S2, WPTS3, and WPT-S4) are the serial numbers of the WPT-S component, and we hereafter call them as WPT-S-U1 and WPT-S-U2 oriented in the $u$-axis and WPT-S-V1 and WPT-S-V2 oriented in the $v$-axis, with respect to the observation axis. Details of the relationship among coordinates defined for the MSC and WPT-S is provided in "Overview" section. The lengths of each MAST and WPT-S are 5 and $15 \mathrm{~m}$, respectively, which are deployed in the satellite spin plane.

Nine scientific instruments are installed in the Arase satellite to comprehensively measure charged particles, DC electric and magnetic fields, and plasma waves (Miyoshi et al. 2012, 2017). The most distinctive part of the Arase satellite is the installation of the software-type wave-particle interaction analyzer (S-WPIA) (Katoh et al. 2018; Hikishima et al. 2018). The S-WPIA is a software module that functions as a "WPIA," an innovative instrument that was originally proposed by Fukuhara et al. (2009). The WPIA measures wave-particle interactions directly and quantitatively by calculating the correlations between particles and waveforms (Katoh et al. 2013; Kitahara and Katoh 2016). To realize the S-WPIA, a huge data storage named mission data recorder (MDR) with a capacity of $32 \mathrm{~GB}$ was installed on board the Arase. The S-WPIA stores raw data measured by the onboard instruments in the MDR to perform correlation analyses between particles and waveforms.

The Plasma Wave Experiment (PWE) is one of instruments on board the Arase satellite to measure plasma waves and DC electric field. The PWE was elaborately designed to obtain maximum scientific outputs in cooperation with the S-WPIA and other scientific instruments. In the present paper, we introduce the PWE specifications and initial results following the launch of Arase. In the following section, we introduce the PWE specifications. Next, we describe the electromagnetic compatibility (EMC) requirements applied to the Arase satellite to guarantee the quality of plasma wave measurements. In the following two sections, we introduce our observation strategy of the PWE and provide some initial results obtained by the PWE, respectively. Finally, we summarize the present study and our findings.

\section{PWE instrumentation}

\section{Overview}

The PWE was developed to measure DC electric field and plasma waves, covering the frequency range from $\mathrm{DC}$ to $10 \mathrm{MHz}$ for electric field and from a few $\mathrm{Hz}$ to $100 \mathrm{kHz}$ for magnetic field. It consists of an orthogonal electric field sensor (WPT; wire probe antenna), a triaxial magnetic sensor (MSC; magnetic search coil), and receivers named electric field detector (EFD), waveform capture 


\section{$\mathbf{a}$}

\section{WPT-S-V1}

(WPT-S3)
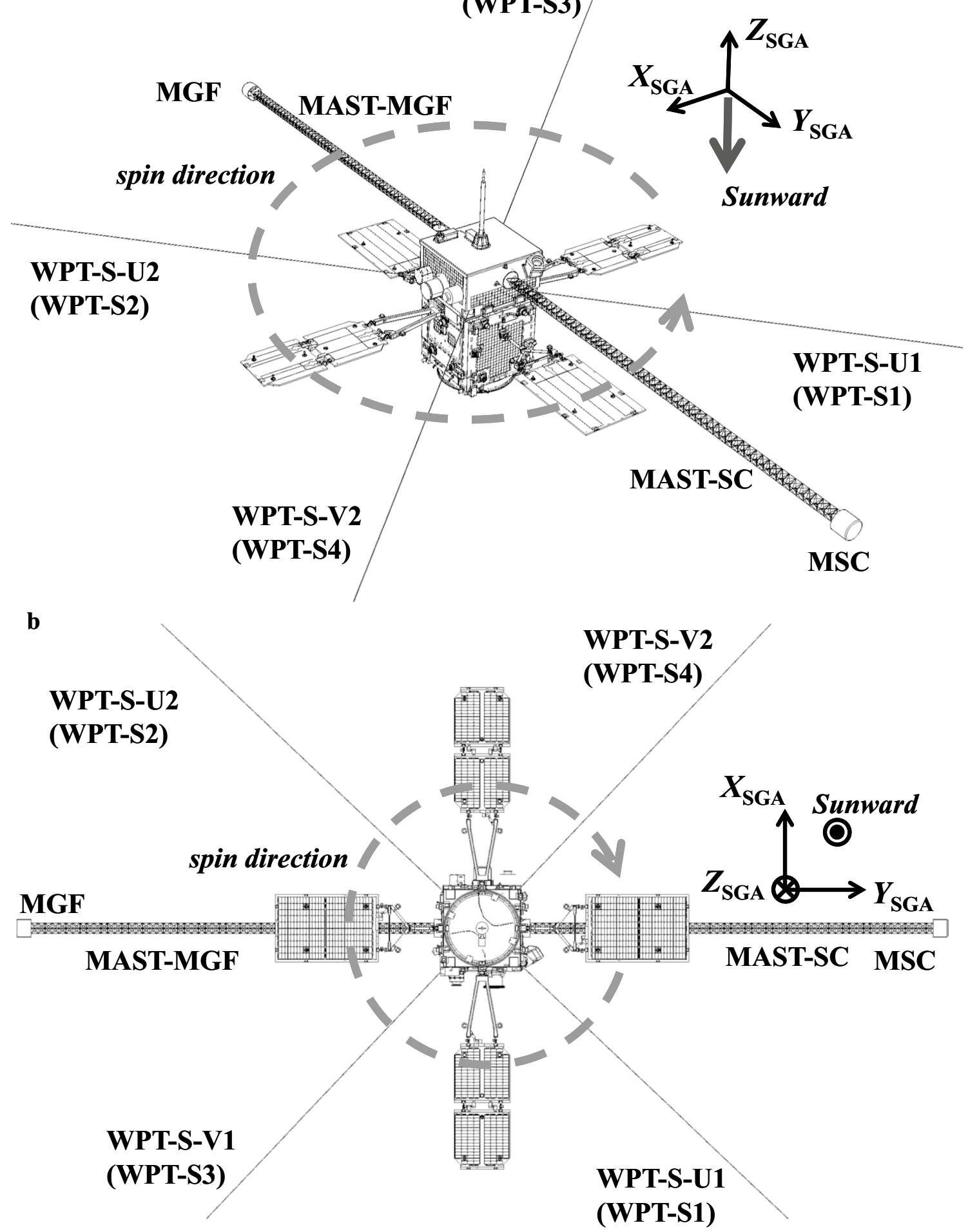

Fig. 1 Overview of the Arase satellite. a External view and $\mathbf{b}$ the view from the $Z_{5 G A}$ direction, where "SGA" stands for "Spinning Satellite Geometry Axis" coordinates, which represent the geometrical positions of the components on board the satellite. The dashed arrows indicate the direction of spacecraft spin motion 
and onboard frequency analyzer (WFC/OFA), and highfrequency analyzer (HFA).

Figure 2a shows the block diagram of the PWE system. The WPT consists of two sets of orthogonal wire antennas dedicated to measuring the electric field. The MSC is the magnetic field sensor mounted on the top plate of the MAST with a length of $5 \mathrm{~m}$ to avoid electromagnetic contamination radiated from the satellite. The PWE-E is the main electronics box and is shown in Fig. 2b. The followings are the main components contained in the PWE-E.

The EWO (EFD/WFC/OFA) is an abbreviation, generated from the initials of the EFD, WFC, and OFA receivers. The EWO-EFD and EWO-WFC/OFA(E) are the receiver components responsible for electric field measurements, and the signals detected by the WPT sensor (WPT-S) are fed into these components through the preamplifiers (WPT-Pre). The EWO-EFD is dedicated to measuring the $\mathrm{DC}$ and very low-frequency electric fields, while the EWO-WFC/OFA(E) is the electric field receiver measuring from $10 \mathrm{~Hz}$ to $100 \mathrm{kHz}$. The EWO-WFC/ $\mathrm{OFA}(\mathrm{B})$ is the magnetic field receiver and the signals from the MSC sensors (MSC-S) are fed into the EWOWFC/OFA(B) through the preamplifiers (MSC-PA). The HFA is the high-frequency electric and magnetic field receiver, into which two electric field components from the WPT-S and one magnetic field component from the MSC-S are fed (Kumamoto et al. 2018). Clock signals called "S-WPIA clock" are distributed from the PWE to the S-WPIA cooperative particle detectors named XEP (Extremely High-Energy Electron Experiments), HEP (High-Energy Electron Experiments) and MEP (MediumEnergy Particle Experiments) (Miyoshi et al. 2017) through three dedicated lines. As the S-WPIA observation requires both high time resolution and high relative time precision among the instruments, the S-WPIA clock is used to guarantee that the relative observation time precision is much better than $10 \mu$ s between the particle detectors and the PWE.

Two independent CPU boards (CPU\#8 and CPU\#9) are installed in the PWE-E. The design of the CPU board is common among the onboard scientific instruments, and ' $\# 8$ ' and '\#9' represent the serial numbers of the CPU boards for the scientific instruments on board the Arase. The CPU\# 8 is responsible for processing signals from the electric field receivers, and the digital data from the EWO-EFD, EWO-WFC/OFA(E) and HFA are fed into the CPU\#8. The CPU\#9 is responsible for processing signals for the magnetic components that are digitized by the EWO-WFC/OFA(B). The CPU boards are mutually connected to form a mission network in the spacecraft. The CPU\#8 and \#9 assigned for the PWE are connected with the MGF (Matsuoka et al. 2018) and the Low-Energy
Particle Experiments (LEP) (Kazama et al. 2017; Asamura et al. 2018), respectively, as shown in Fig. 2a. The mission data processed by these CPU boards are transferred to the mission data processor (MDP), shown on the right in Fig. 2a, through the mission network in the spacecraft (Takashima et al. 2018).

The MWE (MAST/WPT-S Electronics) is the electronics for extension control of the MAST and WPT-S. (The MWE1 is responsible for the extension control of the WPT-S-U1, WPT-S-U2, and the MAST. The MWE2 is responsible for the extension control of the WPT-S-V1 and WPT-S-V2.) The power supply units (PSU) for the instruments as well as extension motors and power distributor (IPD; Instruments power distributor) are also implemented in the PWE-E. The mass and power consumption of the PWE is summarized in Tables 1 and 2.

Figure 3 shows the PWE sensor configurations and the coordinate systems. In this figure, the spinning satellite geometry axis (SGA) coordinates are represented by $X, Y, Z$. In the SGA coordinates, the spin axis of the satellite is defined as the $Z_{\mathrm{SGA}}$-axis, and the MAST-SC is directed toward the positive $Y$-direction. The WPT is radially deployed and directed in the $\pm u$ and $\pm v$ directions. Coordinates $u$ and $v$ are orthogonal and deviate by $133.5^{\circ}$ anticlockwise from the $+X_{\mathrm{SGA}}$ and $+Y_{\mathrm{SGA}}$ directions, respectively. On the other hand, the coordinate systems for the MSC are defined by the symbols $(\alpha, \beta, \gamma)$, where $\alpha$ and $\beta$ deviate by $45^{\circ}$ clockwise from the $+X_{\mathrm{SGA}}$ and $+Y_{\mathrm{SGA}}$ directions, respectively, and $\gamma$ is parallel to the $+Z_{\mathrm{SGA}}$ direction.

The main scientific objectives of the PWE are as follows:

1. To obtain measurements of waveforms and spectra of whistler-mode chorus, MSW, EMIC, etc., and to clarify the mechanisms of acceleration/loss of radiation belt particles through interaction with plasma waves, as well as the generation and propagation characteristics of plasma waves in the inner magnetosphere.

2. To obtain measurements of the DC electric field, which is thought to play an important role in the development of plasma outflow from the plasmasphere during magnetic storms, plume formation, and enhancement of injection processes of ring current particles. It is also important to clarify the generation mechanism of the global DC electric field and its growth process.

3. To obtain measurements of local electron density derived from upper hybrid resonance (UHR) waves with fine frequency and time resolutions. These data will help investigate large-scale fluctuations in the plasmasphere as well as changes of density and tem- 
a WPT

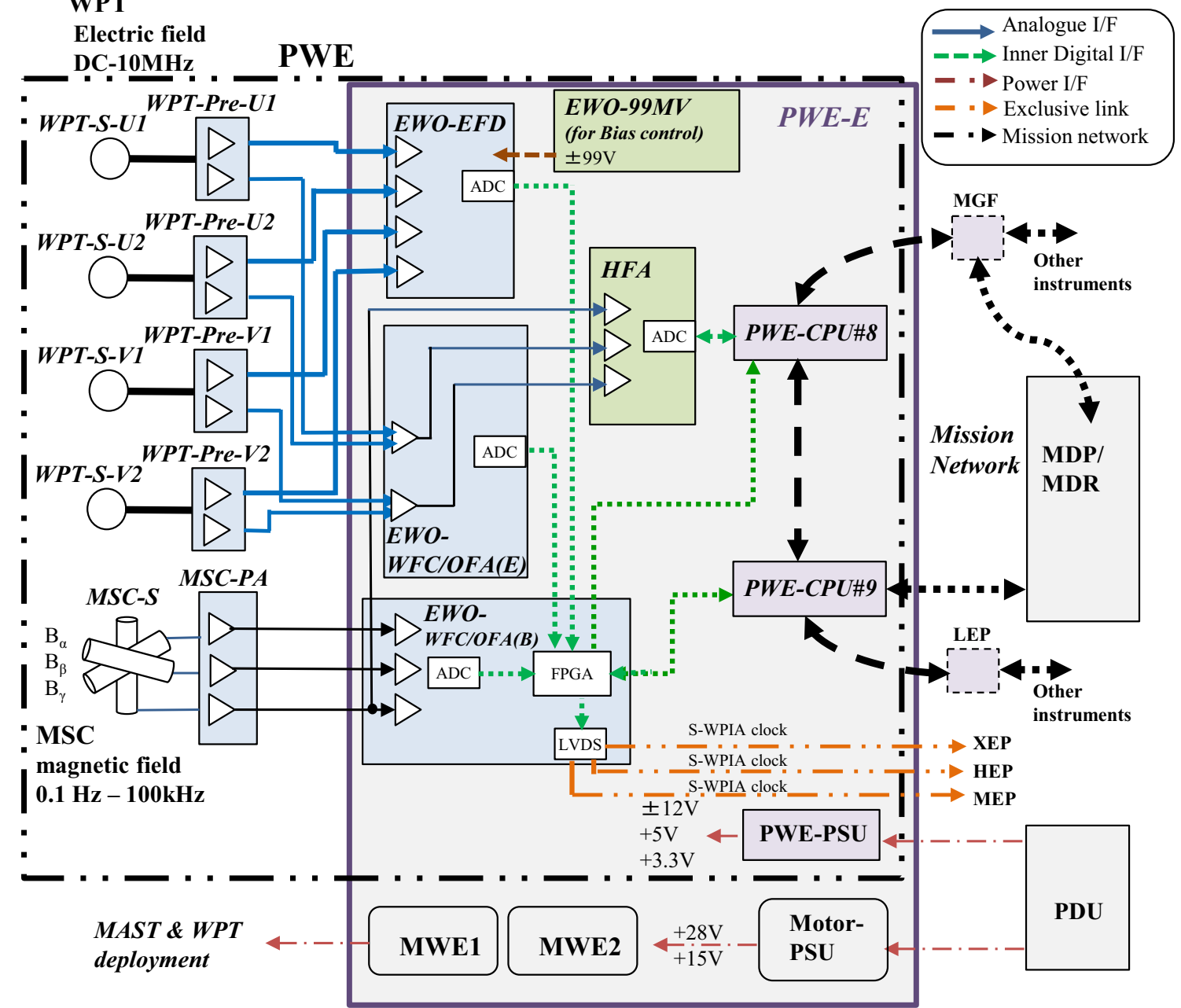

$\mathbf{b}$

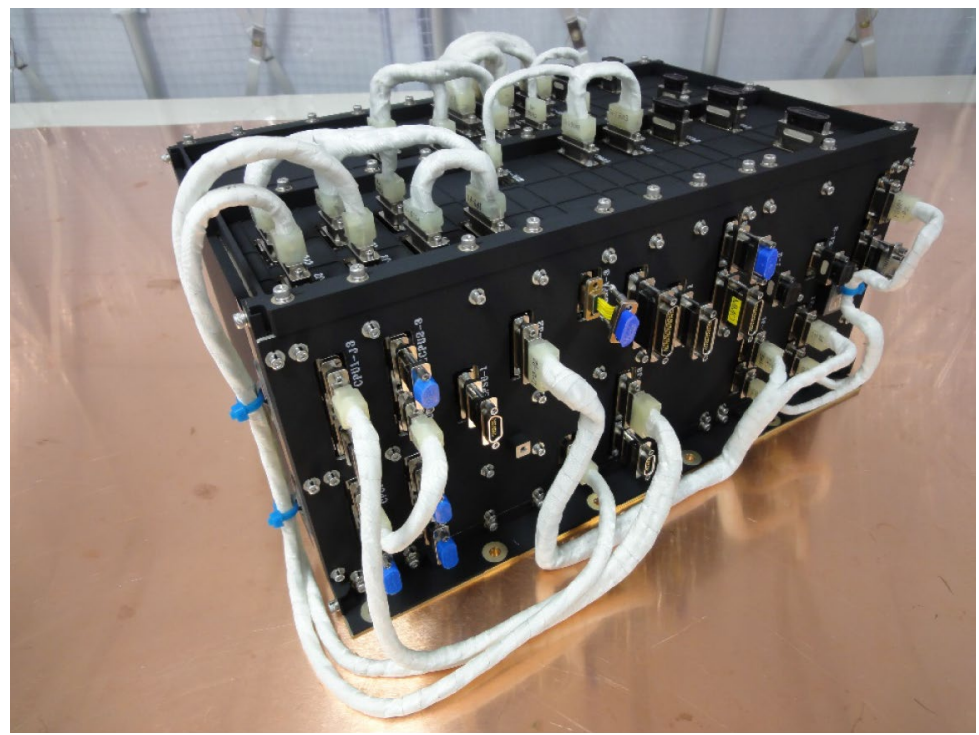

Fig. 2 a Block diagram of the PWE system and $\mathbf{b}$ the main electronics box (PWE-E) 
Table 1 PWE component masses

\begin{tabular}{ll}
\hline PWE (total) & $14.549 \mathrm{~kg}$ \\
PWE-E & $6.335 \mathrm{~kg}$ \\
WPT-PRE & $0.733 \mathrm{~kg}$ (for four units) \\
WPT-S & $3.059 \mathrm{~kg}$ (for four units) \\
MSC-PA & $0.688 \mathrm{~kg}$ \\
MAST-MSC (including the MSC-S) & $3.734 \mathrm{~kg}$
\end{tabular}

Table 2 PWE power consumption

\begin{tabular}{ll}
\hline PWE operation mode & Power (W) \\
\hline CPU ON & 19.00 \\
PWE ON: standby & 30.98 \\
PWE ON: OBS mode (nominal) & 33.29 \\
PWE ON: extension mode (standby) & 38.49 \\
PWE ON: MAST extension & 38.49 \\
PWE ON: WPT-S-V1/N2 extension & 51.17 \\
PWE ON: WPT-S-V3/N4 extension & 51.17 \\
\hline
\end{tabular}

perature profile in the inner magnetosphere during magnetic storms. Another aim is to understand the generation and mode conversion processes of electromagnetic waves.

In addition to the objectives mentioned above, we expect to estimate ion constituents of background plasma derived from characteristic frequencies of EMIC waves in multiple component plasma. As EMIC waves exhibit characteristic frequencies, such as lower hybrid resonance and cutoff frequencies (Matsuda et al. 2014a, b, 2015, 2016), it is possible to estimate constituents of cold ions from these characteristic wave frequencies.

Finally, we show characteristic frequencies and intensities of expected wave phenomena in Fig. 4. The sensitivities and frequency coverage of the electric field measurements using the WPT and the receivers (EFD, WFC/OFA(E), and HFA) are also shown. Figure 5 shows the characteristic frequencies and intensities of

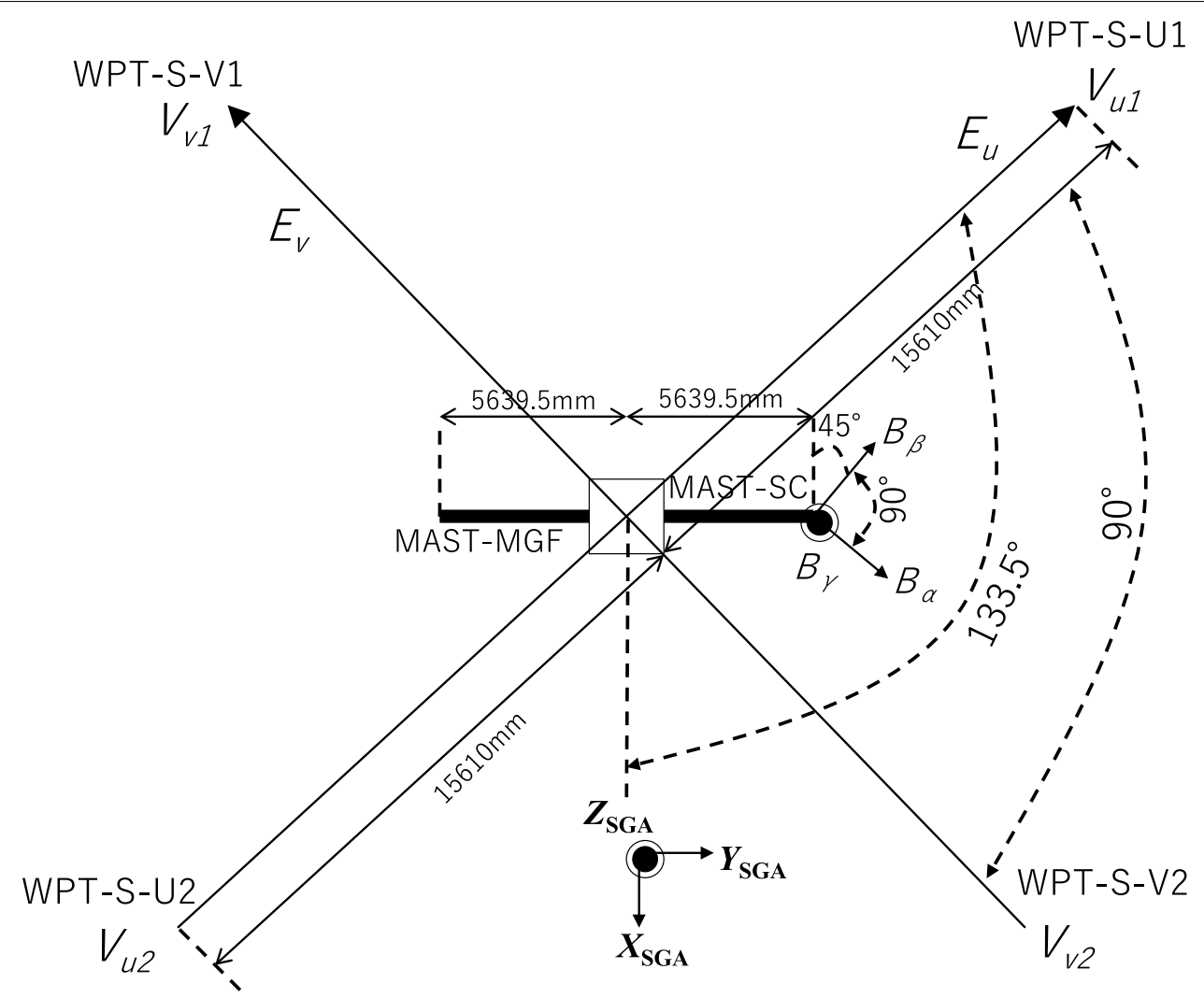

$X_{\mathbf{S G A}}, Y_{\mathbf{S G A}}, Z_{\mathbf{S G A}}$ : SGA Coordinates $\quad u, v$ : Electric field $\quad \alpha, \beta, \gamma$ : Magnetic field

Fig. 3 Configuration of the PWE sensors deployed from the Arase satellite. The SGA coordinates are represented by $X, Y, Z$. The WPT is radially deployed and directed in the $\pm u$ and $\pm v$ directions, and the coordinate systems for the MSC are defined by the symbols $(a, \beta, \gamma)$ 


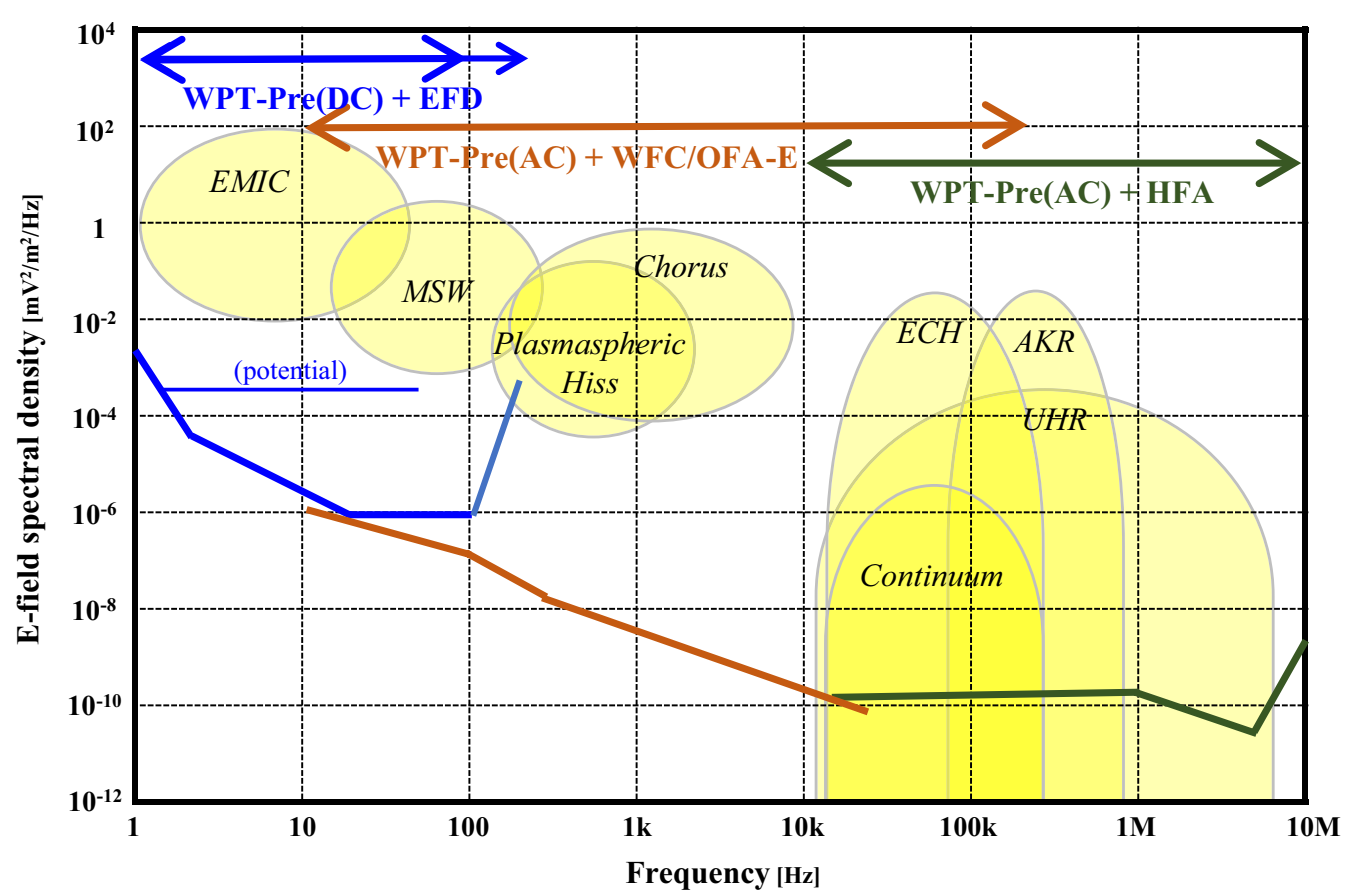

Fig. 4 Electric field wave phenomena as the scientific targets of the ERG mission, and electric field sensor sensitivities and frequency coverage using the WPT and the receivers (EFD, WFC/OFA(E), and HFA)

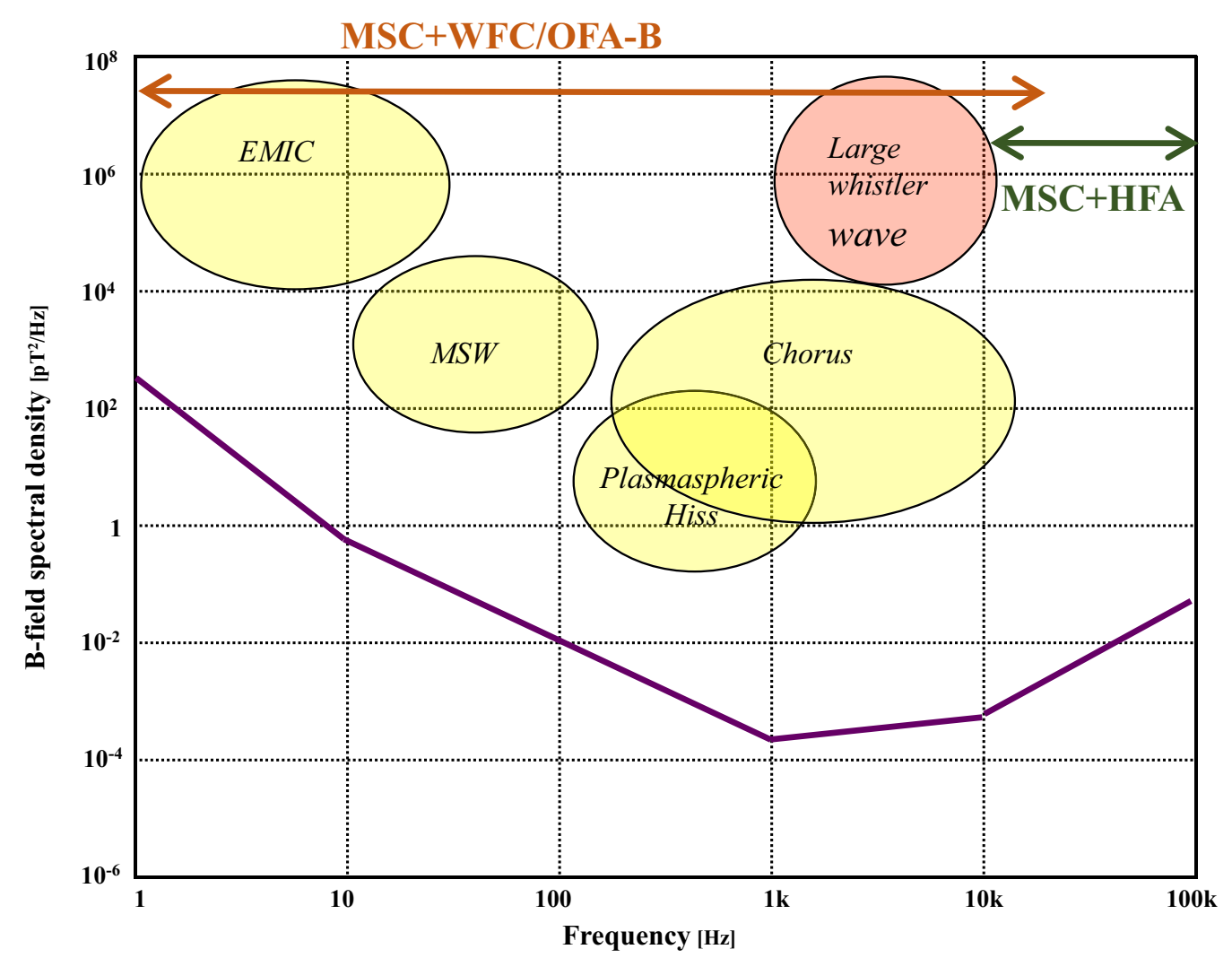

Fig. 5 Magnetic field wave phenomena as scientific targets of the ERG mission, and magnetic field sensor sensitivities and frequency coverage using the MSC and the receivers (WFC/OFA(B), and HFA) 


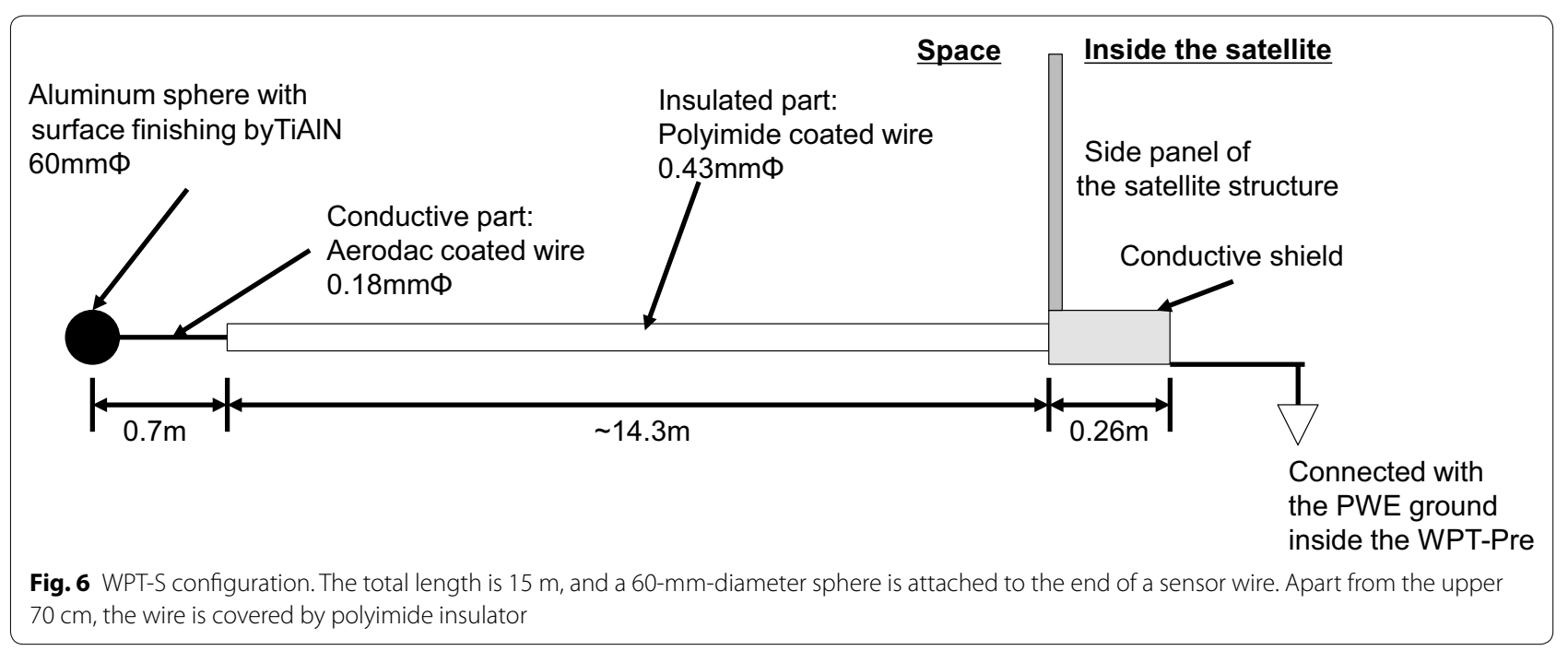

expected wave phenomena as well as the sensitivities and frequency coverage of the magnetic field measurements using the MSC and the receivers (WFC/OFA(B), and HFA). As shown in these figures, the sensitivity and frequency coverage of the PWE allow it to measure all expected plasma waves and radio waves in the inner magnetosphere. In the following subsections, we describe the subcomponents of the PWE.

\section{WPT}

The WPT is designed to pick up the electric field from DC to $10 \mathrm{MHz}$. As shown in Fig. 6, the total length of the WPT is $15 \mathrm{~m}$ (32 m from tip to tip), and a 60-mmdiameter sphere is attached to the end of a sensor wire. Apart from the upper $70 \mathrm{~cm}$, the wire is covered by polyimide insulator. These wires extend orthogonally in the spacecraft spin plane, almost perpendicular to the Sun. This system is similar to the WPT that was developed for the Plasma Wave Investigation (PWI) (Kasaba et al. 2010) on board the BepiColombo Mercury Magnetospheric Orbiter (MMO), except that aluminum alloy was used to reduce the sphere probe mass (as opposed to titanium alloy in the MMO). The detailed specifications and performance of the WPT developed for the Arase are presented in Kasaba et al. (2017).

\section{MSC}

The AC magnetic field vector observations are essential for probing plasma waves. The magnetic field observations are generally performed using search coil magnetometers for low-frequency bands (less than several tens of $\mathrm{kHz}$ ) and loop antennas for high-frequency bands (over $100 \mathrm{kHz}$ ). The Arase satellite carries a triaxial search coil magnetometer to measure the whistler-mode waves below the local electron cyclotron frequency, which is the main target of the mission. The MSC is inherited from the AKEBONO (Fukunishi et al. 1990), GEOTAIL (Matsumoto et al. 1994), and MMO (Kasaba et al. 2010) missions. The MSC on board the Arase was developed based on the design for the future MMO spacecraft because the flight model for the MMO was constructed in advance for the Arase. Figure 7 shows the block diagram of the MSC. The MSC adopts a current-sensitive amplifier (Ozaki et al. 2014) instead of a common voltage amplifier using the flux feedback technique (Matsumoto et al. 1994). The advantages of the current-sensitive amplifier include a lower input impedance and an elimination of the flux feedback coil. The disadvantage is that it consumes more power than the voltage amplifier using the flux feedback, as an equalizer is required to improve the signal dynamic range. This problem was, however, solved by low power applications using application-specific integrated circuits (ASIC) technology (Ozaki et al. 2014, 2016).

Three components of the magnetic field below $20 \mathrm{kHz}$ detected by the MSC are fed to the WFC/OFA(B), and one component of the magnetic field along the satellite spin axis from 10 to $100 \mathrm{kHz}$ is fed to the HFA. Each sensor of the MSC has a gain adjustment amplifier to avoid signal saturation by abnormally large-amplitude whistlermode waves in the radiation belts (Cattell et al. 2008). The saturation levels for high and low gains are 1 and $10 \mathrm{nT}$, respectively, at $1 \mathrm{kHz}$. The EWO-WFC/OFA(B) supplies the gain control signal, squared pulses for calibration, and power line. The MSC covers a frequency range from a few $\mathrm{Hz}$ to $100 \mathrm{kHz}$. The noise equivalent magnetic induction is $20 \mathrm{fT} / \mathrm{Hz}^{1 / 2}$ (femto-Tesla/ $/ \mathrm{Hz}^{1 / 2}$ ) at $1 \mathrm{kHz}$, and the nominal power consumption is $0.43 \mathrm{~W}$ 


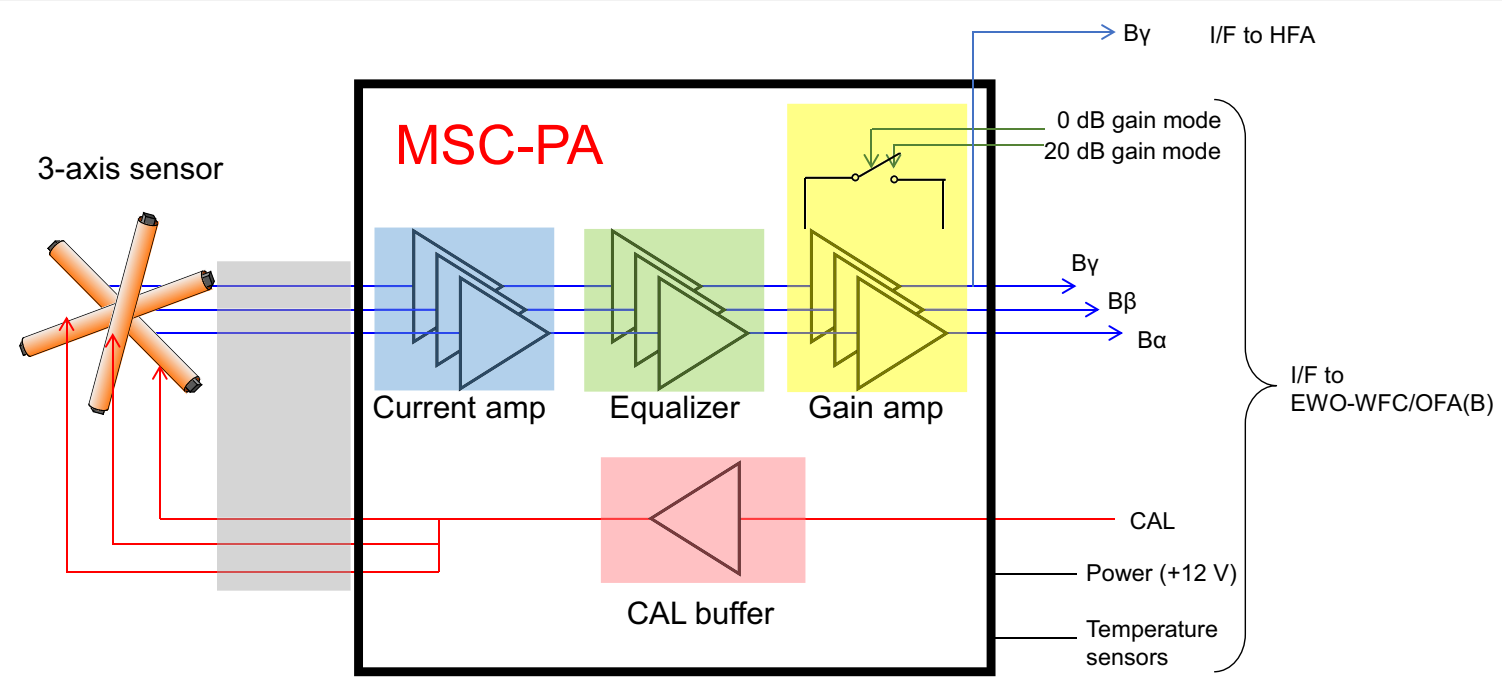

Fig. 7 Simplified block diagram of the MSC sensors and preamplifiers. Three components of the magnetic field below $20 \mathrm{kHz}$ and one component of the magnetic field along the satellite spin axis from 10 to $100 \mathrm{kHz}$ are fed to the WFC/OFA(B) and the HFA, respectively

with a 12-V supply. A detailed description of the MSC performance is available in Ozaki et al. (2018).

\section{EFD}

The EFD measures DC and extremely low-frequency electric field. Two types of measurements, single probe (SPB) and double probe (DPB), are performed by the EFD. As shown in Fig. 3, the SPB provides four channels of satellite potential (i.e., $V_{\mathrm{u} 1}, V_{\mathrm{u} 2}, V_{\mathrm{v} 1}$, and $V_{\mathrm{v} 2}$ ) using the four sets of WPT-S independently at a sampling rate of $128 \mathrm{~Hz}$. The dynamic range of the SPB is $\pm 100 \mathrm{~V}$. The satellite potential is an important diagnostic of electron density in combination with the UHR frequency, measured by the HFA. The DPB provides two channels of differential signals $\left(E_{\mathrm{u}}\right.$ and $E_{\mathrm{v}}$ shown in Fig. 3) that are measured by the two pairs of orthogonal WPT at a $512 \mathrm{~Hz}$ sampling rate. The dynamic range of the DPB is nominally $\pm 200 \mathrm{mV} / \mathrm{m}$, but data with a dynamic range of $\pm 3 \mathrm{~V} / \mathrm{m}$ and a sampling rate of $128 \mathrm{~Hz}$ can be also provided from the SPB output using onboard software. The DPB is dedicated to measuring the DC electric field and ion mode waves, such as EMIC. The waveforms measured by the DPB are expected to be analyzed by combining the magnetic waveforms measured by the MGF (Matsuoka et al. 2018). Detailed specifications and performance of the EFD are presented in Kasaba et al. (2017).

\section{Waveform capture/onboard frequency analyzer}

The WFC and OFA are dedicated to measuring plasma waves between $10 \mathrm{~Hz}$ and $100 \mathrm{kHz}$ for the two electric field components and between $10 \mathrm{~Hz}$ and $20 \mathrm{kHz}$ for the three magnetic field components. The alternative upper frequency bound of 20 and $120 \mathrm{kHz}$ for the electric field components is selected by the telemetry command. The WFC/OFA covers the crucial frequency range, in which chorus waves, hiss waves, and magnetosonic waves appear. The WFC and OFA share the same analog circuits. While the WFC provides the time domain data as waveforms, the OFA outputs digitally processed frequency domain data from the waveforms. Figures 8 and 9 show the block diagrams of the WFC/OFA(E) and WFC/OFA(B), respectively, as well as their sensors and preamplifiers.

\section{Preamplifier for the plasma wave electric field component}

The electric fields picked up by the WPT-S are fed into the WFC/OFA(E) through its preamplifier (WPT-Pre). The PWE has two types of preamplifiers, depending on their frequency range coverage (Kasaba et al. 2017). Figure 10 shows the simplified front-end circuit of the preamplifiers for the electric field measurements. The WPT-Pre (DC) covers the signal at lower frequencies from DC to $\sim 200 \mathrm{~Hz}$, while the WPT-Pre (AC) is dedicated to measuring frequencies above a few Hz. Details of the WPT-Pre (DC) are described by Kasaba et al. (2017). The output of the WPT-Pre (AC) is connected to the WFC/OFA(E) input, which is also shared by the HFA (Kumamoto et al. 2018).

The antenna impedance of the electric field sensor strongly depends on plasma parameters, such as plasma density and temperature, which implies that the impedance varies by satellite location. Since the efficiency of electric field antenna detection is related to its antenna impedance, the input impedance of the preamplifier 


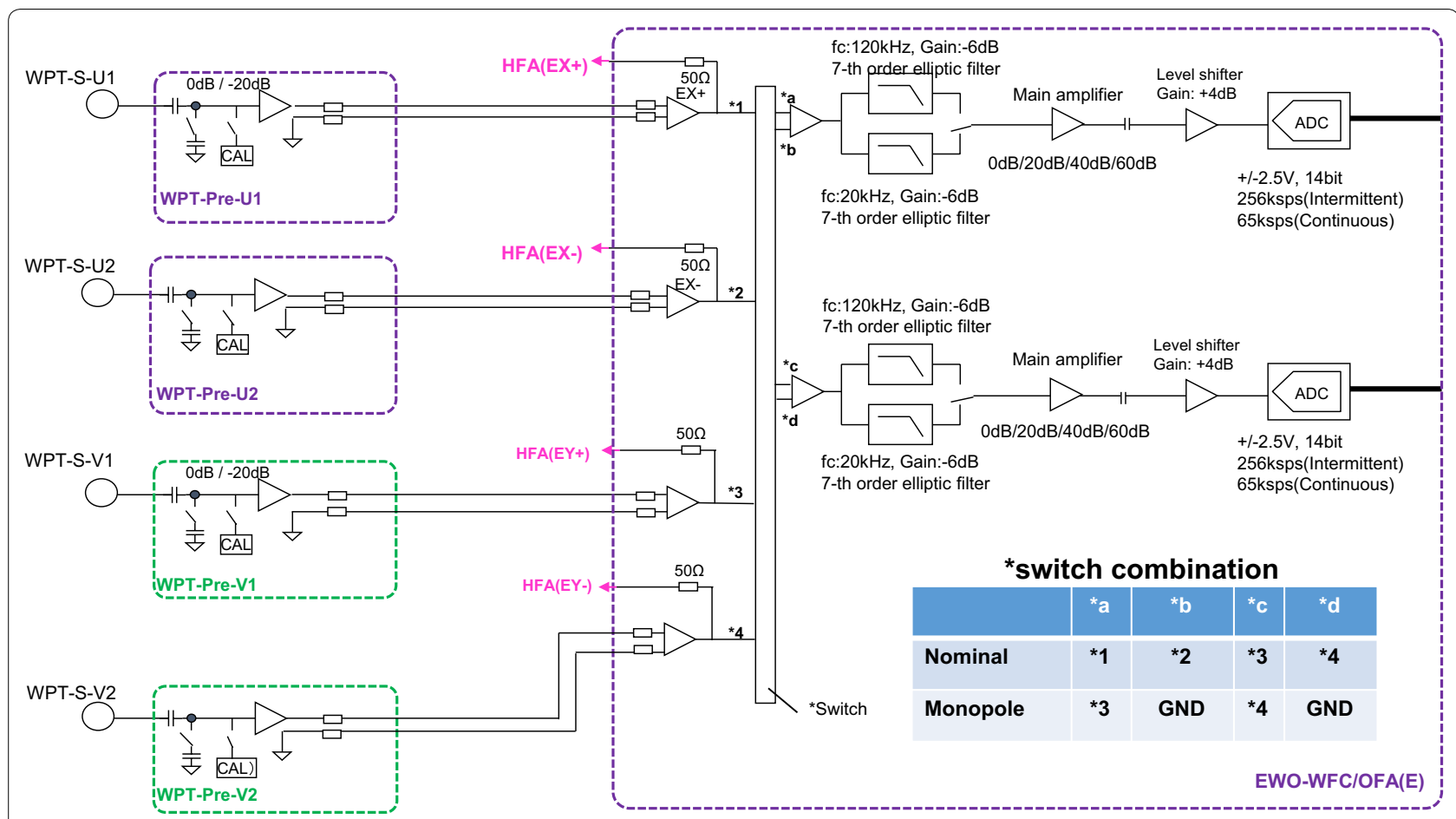

Fig. 8 Simplified block diagram of the WPT and EWO-WFC/OFA(E). Two sets of differential signals between WPT-S-U1 and WPT-S-U2, and WPT-S-V1 and WPT-S-V2 are fed to the WFC/OFA(E) in the "Nominal mode", while the WPT-S-V1 and WPT-S-V2 are connected to each electric field channel of the WFC/OFA(E) and both are used as monopole antennas in the "Monopole mode"

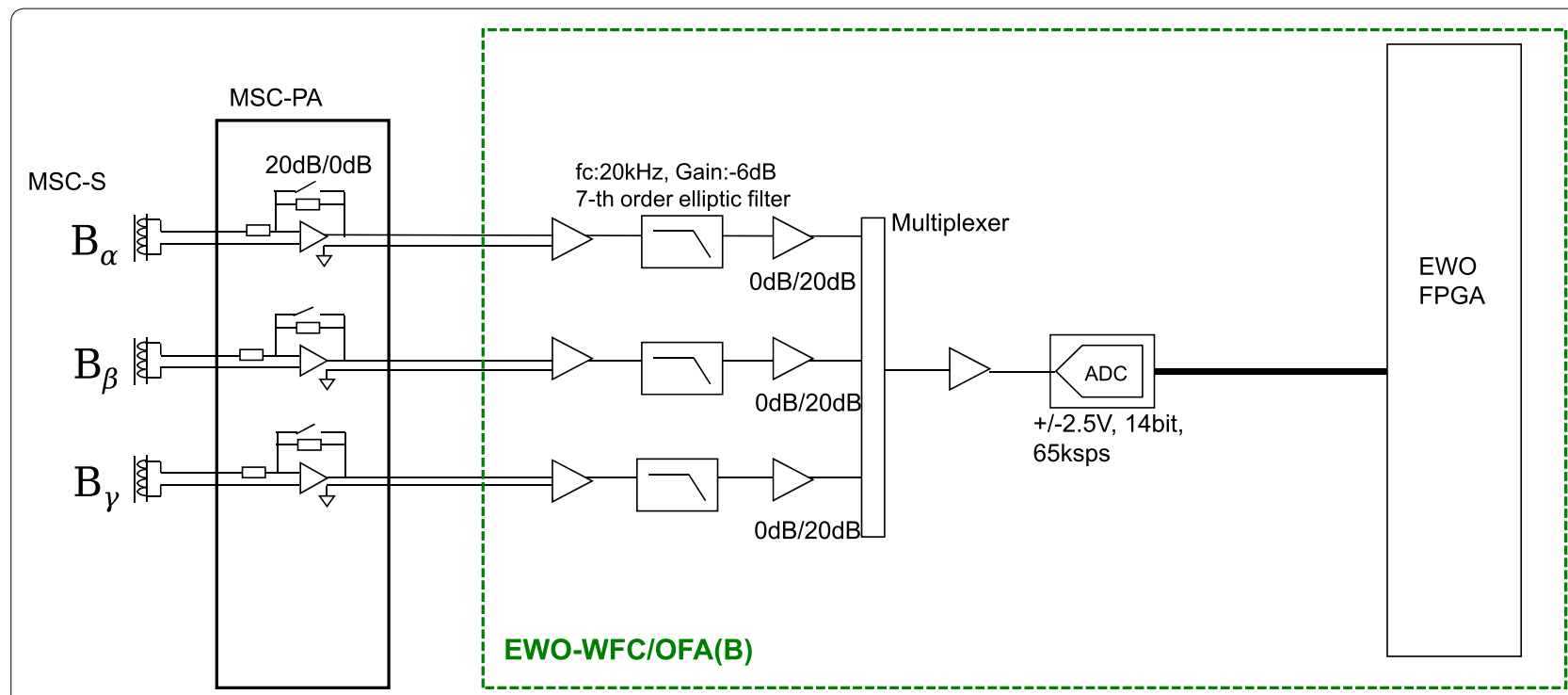

Fig. 9 Simplified block diagram of the MSC and EWO-WFC/OFA(B). Detailed specifications and performance of the MSC and EWO-WFC/OFA(B) are presented in Ozaki et al. (2018)

should be much higher than the expected antenna impedance to pick up electric field component of plasma waves regardless of satellite location. In WPT-Pre (AC) design the input impedance is $220 \mathrm{M} \Omega$. As we adopt a decoupling capacitance of $220 \mathrm{pF}$, a high-pass filter (HPF) with a cutoff frequency of $3 \mathrm{~Hz}$ is formed. The filter eliminates ultra-low-frequency waves as well as the electric field caused by $\mathbf{V} \times \mathbf{B}$, through the spacecraft spin 


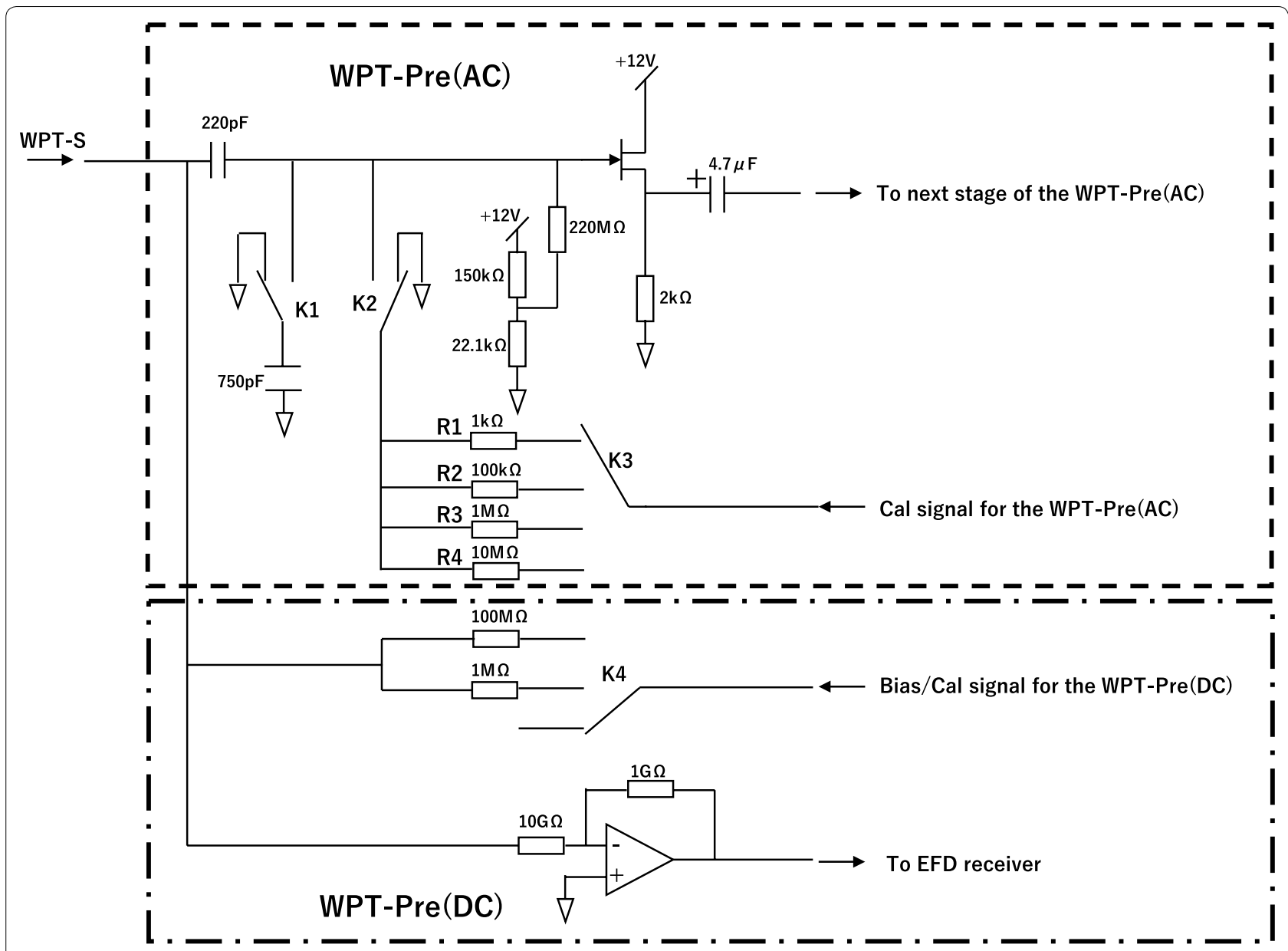

Fig. 10 Simplified circuit of the preamplifier (WPT-Pre) for electric field measurements. The WPT-Pre (DC) covers the signal at lower frequencies from DC to $200 \mathrm{~Hz}$, while the WPT-Pre (AC) is dedicated to measuring frequencies above a few $\mathrm{Hz}$

motion, typically at a frequency of $1 / 8 \mathrm{~Hz}$. Here, $\mathbf{V}$ and B denote the satellite velocity and the ambient magnetic field, respectively.

The WPT-Pre (AC) has an alternative gain control that is selected by the telemetry command. Figure 11 shows the frequency response of the WPT-Pre (AC) in high gain mode. As shown in the upper panel, the WPT-Pre (AC) covers frequencies above a few $10 \mathrm{~Hz}$ at approximately $6 \mathrm{~dB}$ gain. As the loss at the WPT-S input exists in the coupling with its input capacitance, peripheral capacitance, and the antenna impedance, the total gain of the WPT-Pre (AC) accounting for antenna impedance is almost unity. "Onboard calibration system" section describes the equivalent circuit, which considers the coupling of the WPT-Pre (AC) input and the WPT-S.

The attenuation at the WPT-Pre (AC) input is effective by turning on the K1 switch (Fig. 10) in low-gain mode. The 750-pF capacitance attenuates the detected plasma waves in combination with the antenna impedance. This means that the precise attenuation level primarily depends on the ratio of the antenna impedance to the 750-pF capacitance. Using the onboard calibration system, described in "Onboard calibration system" section, the precise preamplifier gain in low-gain mode is measured.

The precise electric field sensitivity, displayed in Fig. 4, can be obtained from the flight data of the WPT-Pre (AC) after launch. The sensitivity shown in Fig. 4 is obtained by assuming $70 \mathrm{pF}, 10 \mathrm{M} \Omega$ and $15 \mathrm{~m}$ for the theoretical antenna capacitance in vacuum, typical plasma impedance, and the effective length of the WPT-S, respectively.

The identification of the electric field antenna impedance is necessary to calibrate the observed electric field data. In particular, since waveform data contain the phase information, the precise phase calibration (considering the antenna impedance) using the PWE onboard calibration system is very important. The system inputs known waveforms at the WPT-Pre (AC) input through different resistances (R1, R2, R3, and R4 shown in Fig. 10). The 


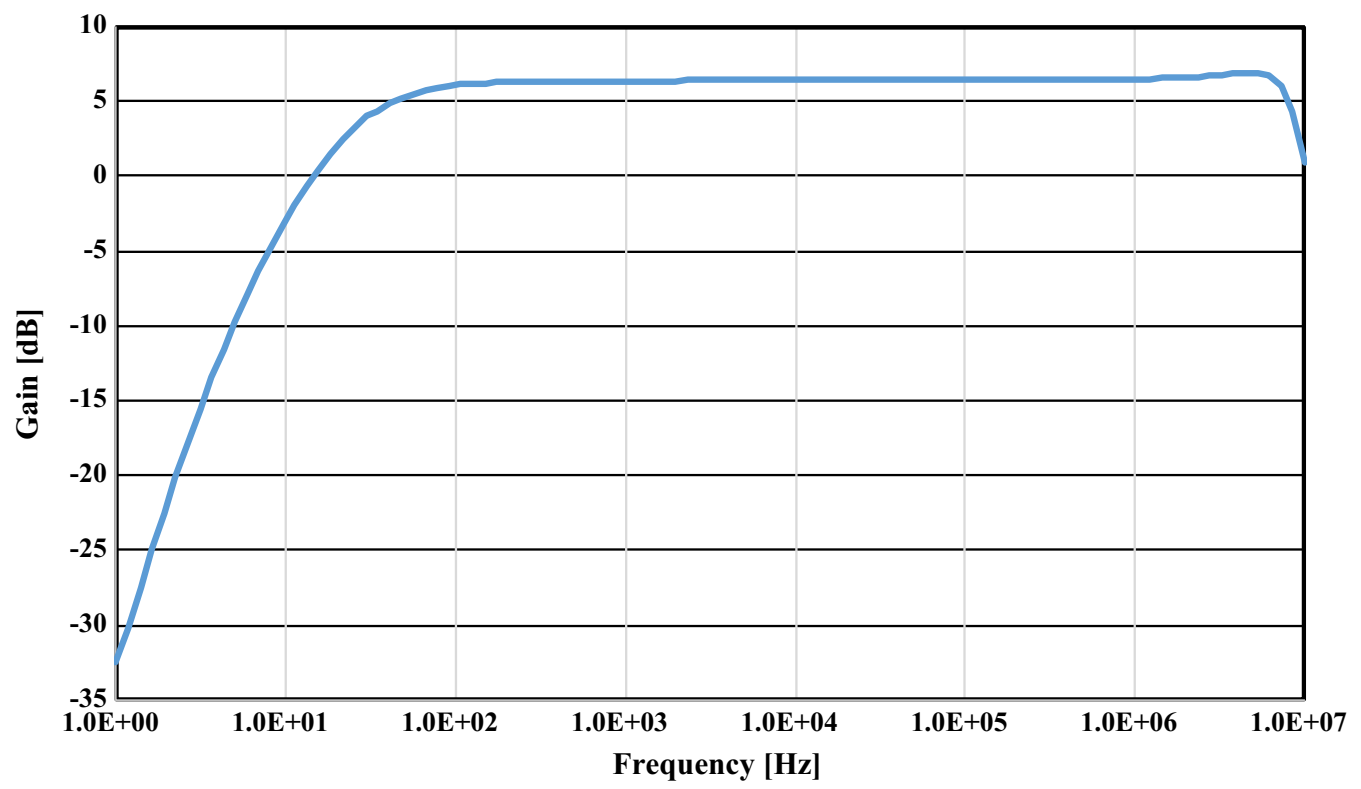

Fig. 11 Frequency response of the WPT-Pre(AC) measured in the ground tests

detailed function of the onboard calibration system is described in "Onboard calibration system" section.

\section{Main receivers}

As shown in Figs. 8 and 9, each channel of the WFC/ OFA consists of a differential amplifier, a low-pass filter (LPF) as the antialiasing filter, a main amplifier, an A/D converter, and a digital processing part. The LPF is the seventh-order elliptic filter that avoids the aliasing effect at the $\mathrm{A} / \mathrm{D}$ converter.

For the electric field channels (Fig. 8), two sets of the LPF with different cutoff frequencies are installed. These cutoff frequencies are 20 and $120 \mathrm{kHz}$, and can be selected by the telemetry command. In the nominal operation, $20 \mathrm{kHz}$ is used for the cutoff LPF value. For the magnetic field channel, one LPF with a $20-\mathrm{kHz}$ cutoff frequency is used.

The gains of the main amplifier can be controlled in four steps for electric field channels and in two steps for magnetic field channels. Figures 12 and 13 show the frequency responses of the WFC/OFA for electric and magnetic field channels, respectively. As shown in these figures, the gain of the electric field channels varies from 0 to $60 \mathrm{~dB}$, while the magnetic field channel gains vary from 0 to $20 \mathrm{~dB}$ by $20 \mathrm{~dB}$ step. The gain change is controlled by the telemetry command and the onboard software. The onboard software has an Auto Gain Control (AGC) function, which controls the WFC/OFA gain considering the received plasma wave intensities (Matsuda et al. 2018).
The WFC directly samples waveforms detected by sensors. While either a 65.536 or $262.144 \mathrm{kHz}$ sampling rate is selected for the electric field component channels by the telemetry command, the sampling rate for the magnetic field component channels is fixed at $65.536 \mathrm{kHz}$. Since these sampling clocks are generated by dividing the common source oscillator with its frequency at 16.777216 MHz, synchronization is guaranteed in the sampling of all WFC/OFA channels. The simultaneous sampling through all WFC/OFA channels guarantees the precision of the relative phase difference between different channels, enabling us examine plasma wave properties, such as polarity and Poynting flux.

The WFC/OFA(E) includes a special mode called "Monopole mode." In this mode, the WPT-S-V1 and WPT-S-V2 are connected to each electric field channel of the WFC/OFA(E) and both are used as monopole antennas. This configuration realizes the "Interferometry mode," which can identify phase difference between the waves detected by the WPT-S-V1 and WPT-S-V2.

\section{HFA}

The signals from the WPT-Pre (AC) are fed to the HFA via the differential buffer amplifiers in the EWO-WFC/ OFA(E). In addition, the signal from the MSC-PA is fed directly to the HFA, which consists of two channels of analog receiver (HFA-A) and digital circuit (HFA-D) for signal processing. In the HFA-A, two signals from two electric field sensors (i.e., $E_{\mathrm{u}}$ and $E_{\mathrm{v}}$ ) and one magnetic field sensor $\left(B_{\gamma}\right)$ are selected and fed to two receiver 


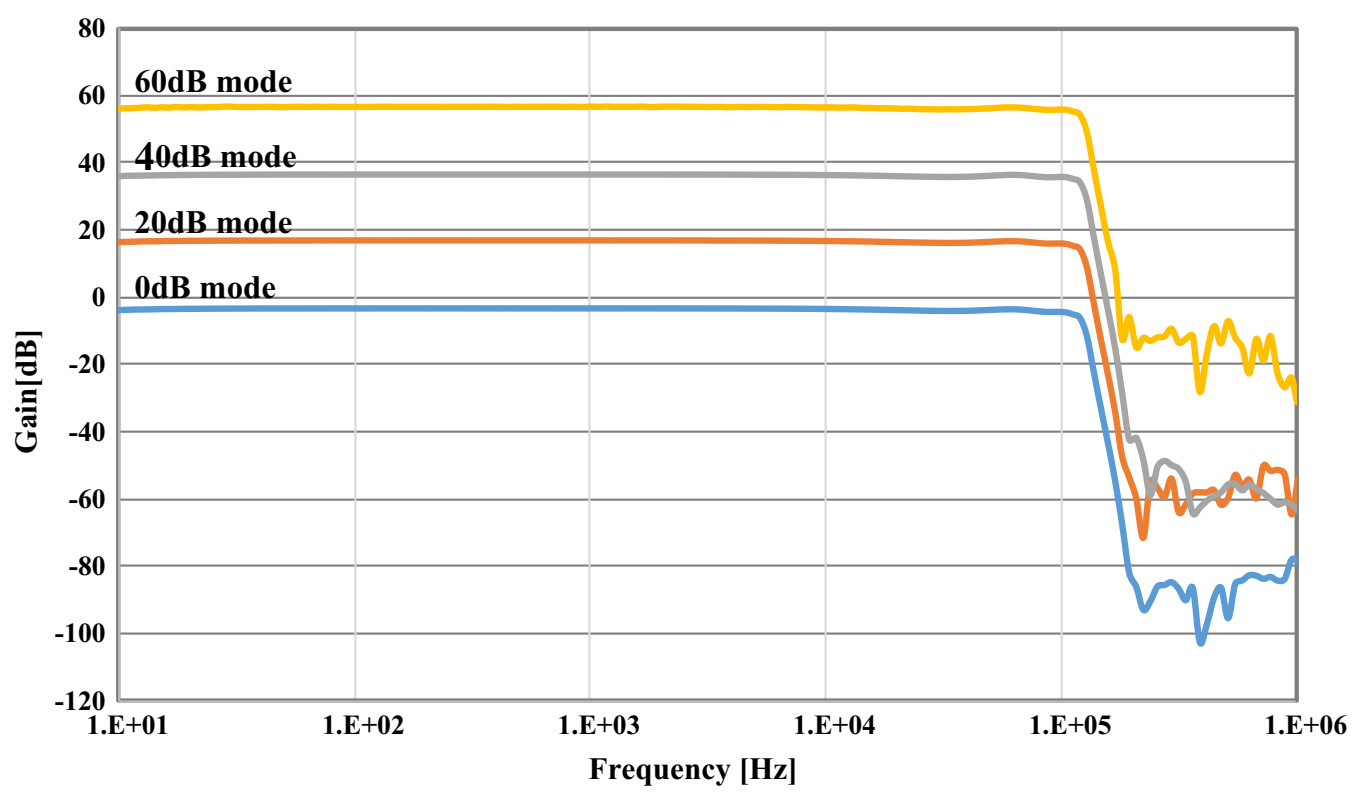

Fig. 12 Frequency response of the WFC/OFA(E) measured in the ground tests

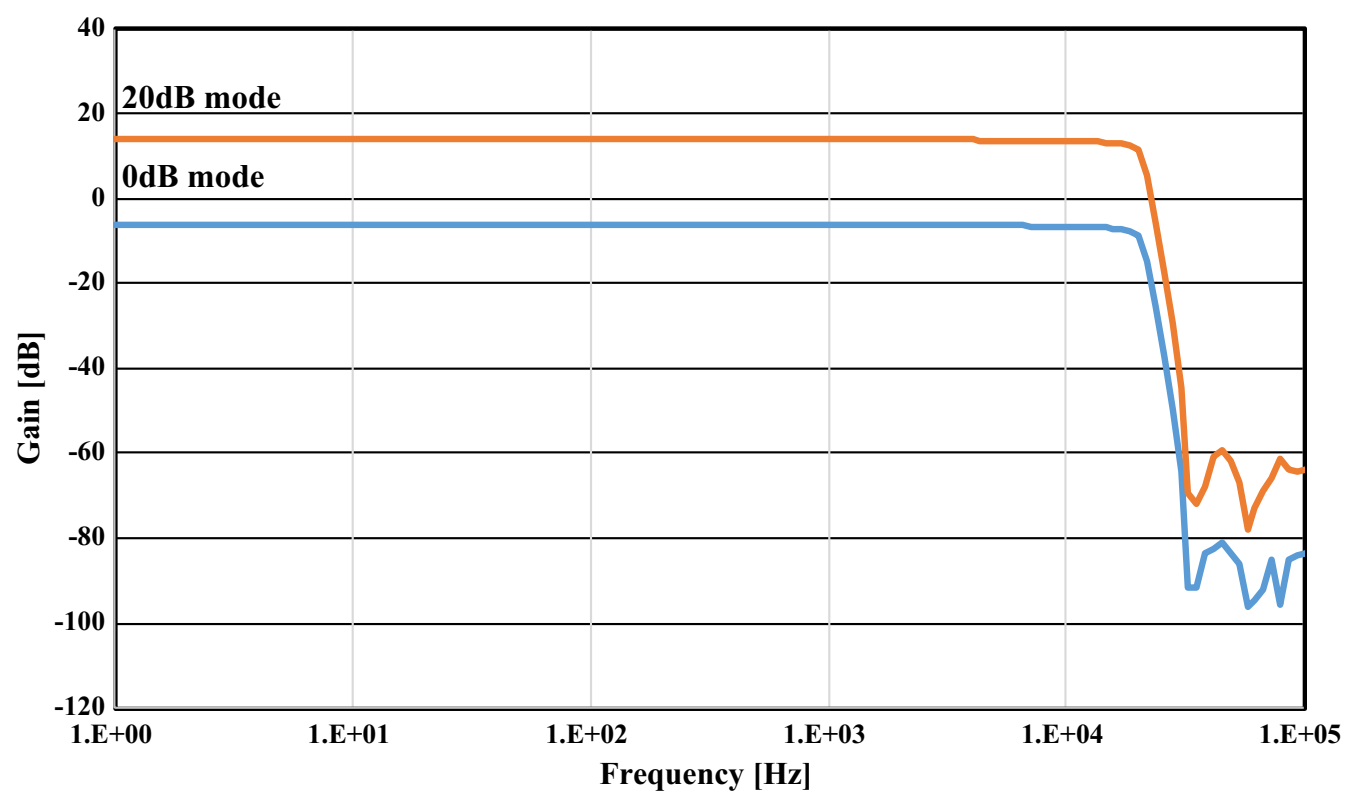

Fig. 13 Frequency response of the WFC/OFA(B) measured in the ground tests

channels. The receivers consist of attenuators $(0$ or $10 \mathrm{~dB}), \mathrm{HPF}$, and LPF. The passband of the receiver is from $10 \mathrm{kHz}$ to $10 \mathrm{MHz}$. The analog signals are sampled by an A/D converter in the HFA-D with a $25-\mathrm{MHz}$ sampling rate. The spectra are obtained in two signal processing modes by field-programmable gate array (FPGA) in HFA-D. In the $10-\mathrm{MHz}$ mode, a fast Fourier transform
(FFT) is directly applied to the 2048 digital waveform data points. In the $1-\mathrm{MHz}$ mode, a cascade integratorcomb (CIC) filter with a passband below $1 \mathrm{MHz}$ and decimation are applied to the signal from A/D converter, resulting in waveform data at 2.5 mega-samples per second (MSPS). FFT is applied to the 2048 points of the 2.5 MSPS waveform. Detailed specifications including a 
block diagram of the HFA are presented in Kumamoto et al. (2018).

\section{Onboard calibration system}

The WFC/OFA, including the WPT-S and MSC-S sensors and their preamplifiers, has an onboard calibration function that measures the frequency responses of sensors and receivers by applying the standard signals generated by the known signal source. The onboard calibration system is used to examine the degradation of sensors and receivers. The signal source we use is the main clock $(16.777216 \mathrm{MHz})$ of the WFC/OFA digital parts and is divided by the FPGA. The divided signal is fed to both the WPT-Pre input and the calibration coil wound inside of the MSC (Ozaki et al. 2018). As the waveform of the calibration signal is rectangular, it consists of odd harmonics, and the waveform covers the wide frequency range. The fundamental frequency of the rectangular waveform can be altered by changing the frequency-dividing rate, which in turn can change by telemetry commands.

The onboard calibration for the MSC is described in Ozaki et al. (2018). Here, we describe the detailed calibration of the electric field sensor with the WPT-Pre (AC) using the onboard calibration system. Identifying the complex impedance of the electric field sensors in plasma is essential to calibrate the observed electric field components of plasma waves. The antenna impedance of the electric field sensor strongly depends on the surrounding plasma environment. In particular, the antenna impedance is sensitive to the plasma densities and temperatures. Since the efficiency in detecting electric fields depends on the antenna impedance, the precise value of the antenna impedance is necessary to calibrate the electric field data. Figure 14 shows the equivalent circuit including the front end of the WPT-Pre (AC). Here, $Z_{\mathrm{A}}$ is the antenna impedance and $Z_{\mathrm{W}}$ is the impedance of the antenna wire, including the inductance and resistance of the wire. $Z_{\mathrm{S}}$ and $Z_{\mathrm{C}}$ are the shield and cable capacities, respectively, between the WPT-S and preamplifiers. $Z_{\mathrm{D}}$ and $Z_{\mathrm{I}}$ are the decoupling impedance and the input capacitance, respectively, of the WPT-Pre (AC).

The calibration signal is fed into the WPT-Pre (AC) through the specific resistance as the output impedance $(K)$ of the calibration signal. The fed calibration signal is affected by the combined impedance $\left(Z_{\mathrm{T}}\right)$ of $Z_{\mathrm{A}}, Z_{\mathrm{W}}, Z_{\mathrm{S}}$, $Z_{\mathrm{C}}, Z_{\mathrm{D}}$, and $Z_{\mathrm{I}} . Z_{\mathrm{T}}$ is defined as

$$
Z_{\mathrm{T}}(f)=\left[\left\{\left(Z_{\mathrm{A}}+Z_{\mathrm{W}}\right)\left\|Z_{\mathrm{S}}\right\| Z_{\mathrm{C}}\right\}+Z_{\mathrm{D}}\right] \| Z_{\mathrm{I}},
$$

where || represents the combined impedance connected in parallel. The calibration signal is divided by its output impedance $(K)$ and $Z_{\mathrm{T}}$. As the input impedance of the WPT-Pre $(\mathrm{AC})$ is large enough, the input voltage $\left(V_{\mathrm{I}}\right)$ of the calibration signal $\left(V_{\mathrm{C}}\right)$ is described as

$$
V_{\mathrm{I}}=\frac{Z_{\mathrm{T}}}{Z_{\mathrm{T}}+K} V_{\mathrm{C}}
$$

The output impedance $(K)$ is selected by the telemetry command among four different resistances of $1,100 \mathrm{k} \Omega$,

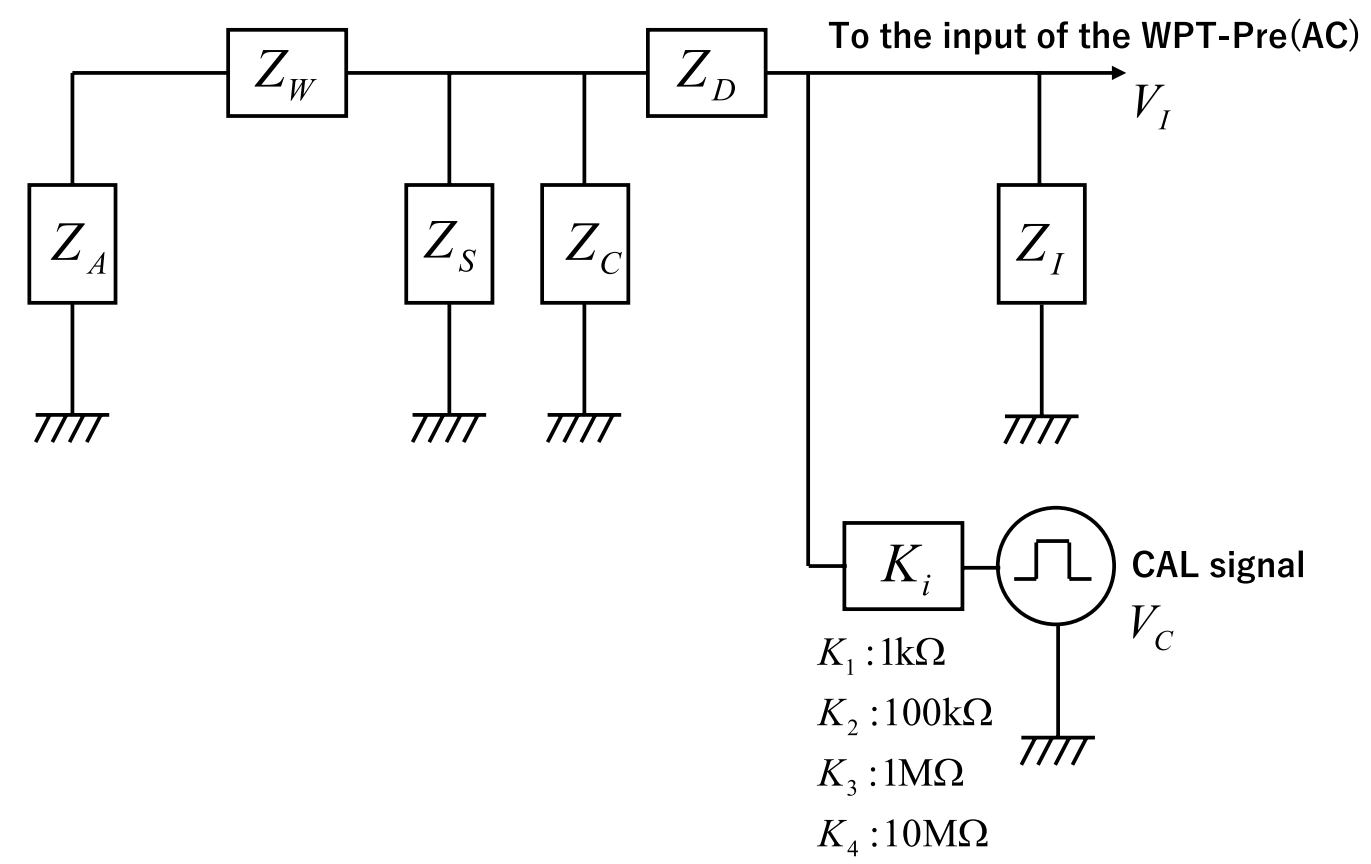

Fig. 14 Equivalent circuit of the calibration system for electric field measurements 
1, and $100 \mathrm{M} \Omega$ (Fig. 14). The divided calibration signal is measured as the waveform through the WFC(E) receiver. Since the original waveform of the calibration signal is known, the complex transfer function $\left(G_{\mathrm{WFC}}(f)\right)$ is obtained by correlating the measured waveform with the original waveform. Here $G_{\mathrm{WFC}}(f)$ includes the effects of $Z_{\mathrm{T}}$ and $K$. However, when the output impedance $K$ is much smaller than $Z_{\mathrm{T}}$, the effect of $Z_{\mathrm{T}}$ is negligible and the calculated $G_{W F C}(f)$ is equivalent to the end-to-end transfer function of the WPT-Pre (AC) and the WFC/ OFA(E). In this instance, the output impedance of $1 \mathrm{k} \Omega$ is used in the end-to-end calibration of the electric field waveform observation. Other output impedances are used for deriving the antenna impedance $Z_{\mathrm{A}}$ from $Z_{\mathrm{T}}$. Since the impedances other than $Z_{\mathrm{A}}$ are known in advance, we can derive $Z_{\mathrm{A}}$ using the obtained transfer function $G_{\text {WFC }}(f)$ by changing the resistances of $K$. The output resistance $K$ and frequencies of the calibration signals be altered by the telemetry command and onboard software. The detailed sequence of onboard calibration and antenna impedance measurement can be found in Matsuda et al. (2018).

\section{CPU}

The CPU board is responsible for the digital processing and consists of the CPU, the SDRAM of $128 \mathrm{MB}$, spacewire communication devices, and their peripheral circuits (i.e., ECSS-E-ST-50-12C 2008; ECSS-E-ST-50-51C 2010; ECSS-E-ST-50-52C 2010). The CPU is the newly developed SOI-Soc chip (silicon-on-insulator-systemon-chip) (Kuroda et al. Kuroda 2011). Through the CPU board, the PWE participates in the spacewire network, connected to all of the scientific instruments, and communicates with other instruments as well as the MDR.

The PWE-E has two independent CPU boards (CPU\#8 and CPU\#9). The data from EWO-EFD, EWO-WFC/ OFA(E), and HFA are sent to CPU\# 8 and the data from the EWO-OFA/WFC(B) are sent to CPU\#9 using spacewire cables. Commands to the receivers and sensors are sent from these CPU boards, and housekeeping and mission data of the PWE are sent to the CPU boards. The onboard software running on these CPU boards provides the FFT calculation function for the OFA and the data compression for the WFC (Matsuda et al. 2018).

Data from the PWE are sent to the CPU boards in units of blocks per second, and these data are stored in the ring buffer on the CPU board. There are two kinds of ring buffers, "Long buffer" and "Short buffer." The maximum recording times stored in these buffers are $\sim 200$ and $\sim 16 \mathrm{~s}$ in the long and short buffers, respectively. The long buffer is primary used in the EFD (except the raw waveform data), OFA, and HFA data processing, which are continuously generated when the PWE is in observation mode. On the other hand, the data stored in the long buffer are frozen during the WFC data processing and the subsequent measured data are sent to the short buffer, as it is necessary to apply a time-consuming (non-real-time) processes, such as data compression or digital filtering to the WFC data. We, therefore, perform the WFC data processing as a background process using the data frozen in the long buffer, while continuing to process the EFD (except the raw waveform data), OFA, and HFA data using the subsequent data sent to the short buffer in parallel. After the WFC data processing is finished, the data flow is switched back to the long buffer. As a result, the WFC data are intermittently generated, while the other data are continuously produced without any data gaps. We show the onboard data processing details in "Features of the PWE functions" section and further specifications of the onboard software are presented in Matsuda et al. (2018).

\section{Electromagnetic compatibility}

The concept of EMC is essential to guarantying the PWE data quality. The Arase mission introduces the EMC concept with respect to the critical measurements of plasma waves and ambient magnetic fields. The serious contamination to the PWE as "noise" degrades the data quality and the EMC requirements with regard to the PWE aim to avoid contamination from other onboard instruments. Note that we do not describe the EMC requirements with regard to the ambient magnetic field measurements in this paper. The noise from onboard instruments is classified into three categories, which include (1) conducted (CE), (2) radiated electric field (RE02), and (3) radiated magnetic field emissions (RE04). The Arase EMC requirements are introduced according to the above three categories. Figures 15, 16, and 17 represent the ERG EMC requirements of the CE, RE02, and RE04, respectively. Each onboard instrument and subsystem are requested to suppress their noise levels below the specified category thresholds.

Figure 15 shows the limit level of the conducted emission, which is defined as the noise on the power line of each instrument. As shown in this figure, there are two limit levels, which signify the normal and the common mode noises. The normal mode noise is the noise that is directly emitted on a hot or return power line. Since all of the onboard instruments operate using the same power supply system, the conducted emissions, as the normal mode, propagate along the PWE power line and contaminate the noise in the PWE observations. The common mode noise is emitted by the noise current due to the unbalanced current, which has the potential to radiate electric field noise through the specific impedance, such as the impedance of the satellite structure. 


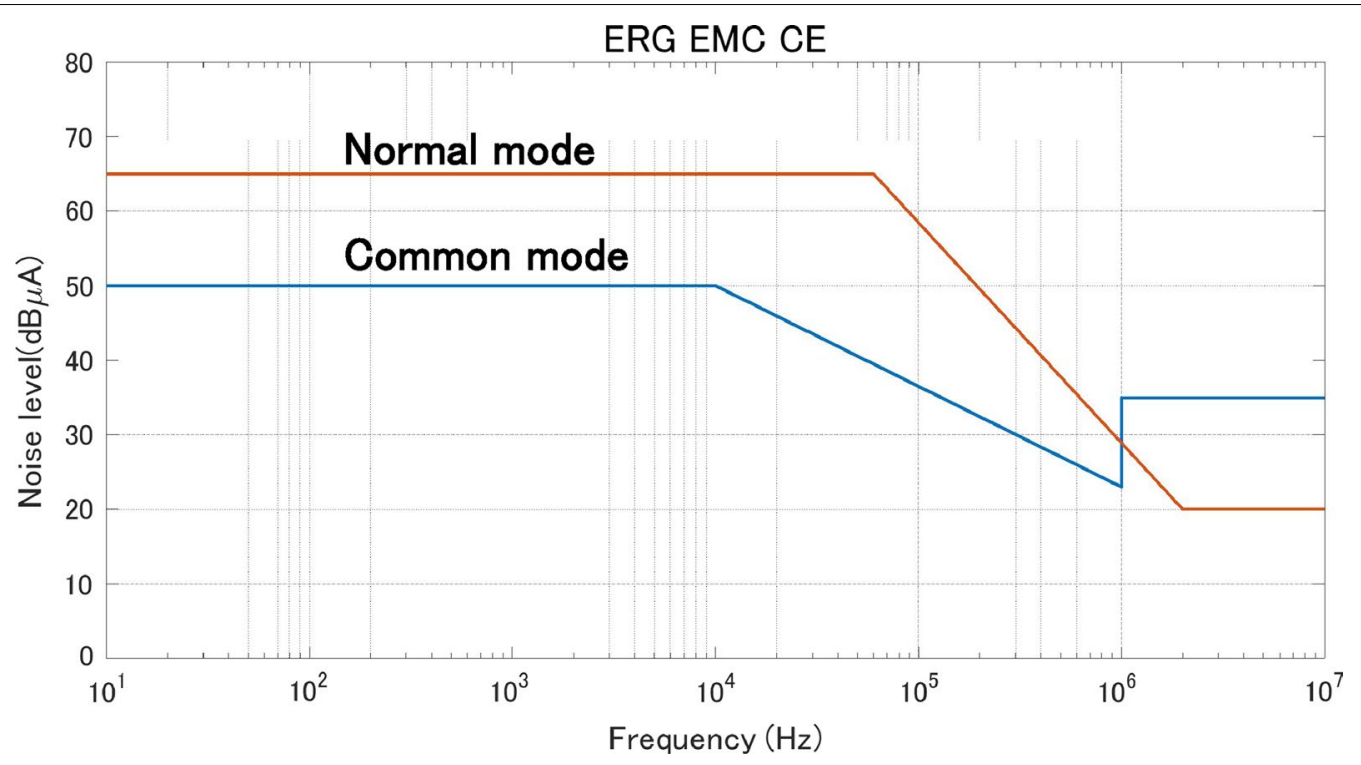

Fig. 15 Limit level for the electromagnetic compatibility (EMC) requirements of the conducted emission (CE)

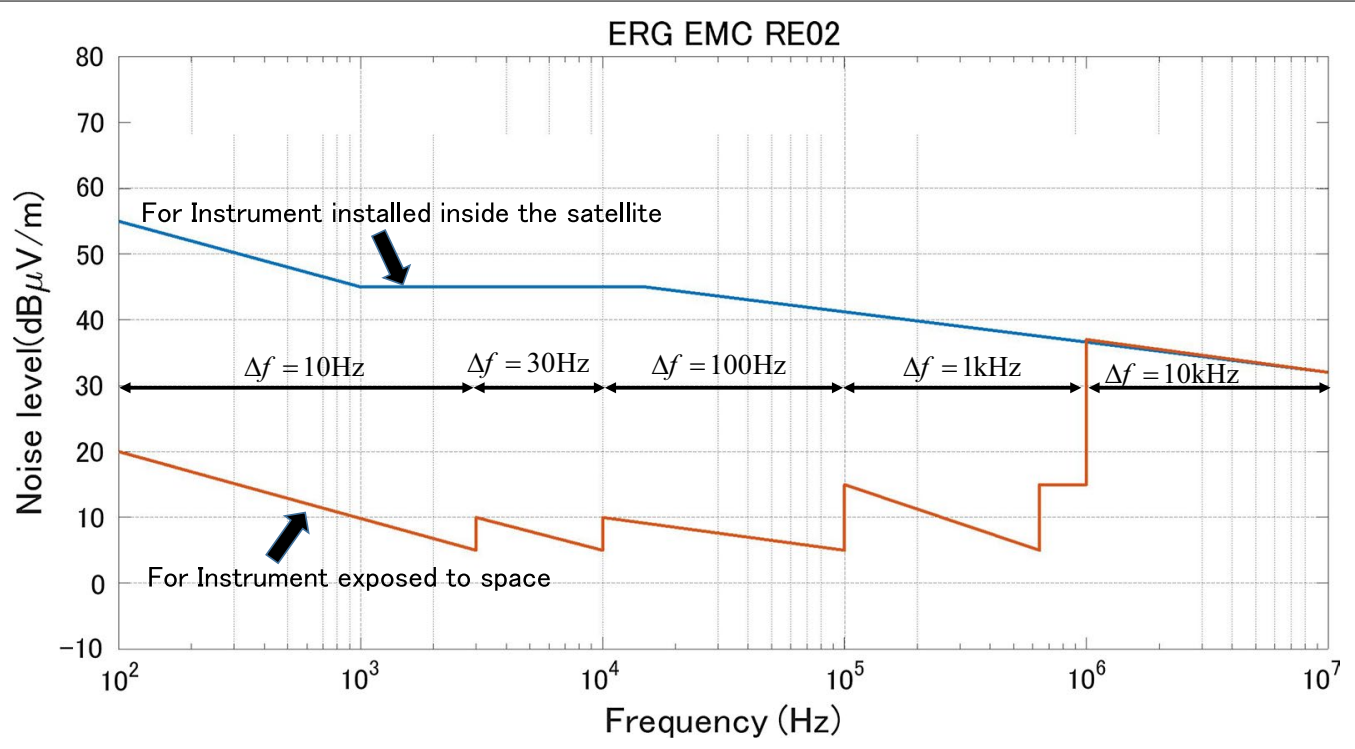

Fig. 16 Limit level for the electromagnetic compatibility (EMC) requirements of the radiated electric field noise (RE02)

Figures 16 and 17 show the limit levels of the radiated electric field noise and radiated magnetic field noise, respectively. $\Delta f$ in each figure denotes the frequency bandwidth used in the noise measurements. By referring to the sensor sensitivities and the specifications of the PWE receivers, we defined the limit levels as the levels at a 1-m distance from each instrument or a subsystem. The two limit levels shown in Fig. 16 were determined by considering the shielding effect of the satellite body. Since the shield effect, due to the satellite panels, is expected for the electric field noise, the limit level is higher for instruments installed inside the satellite than that for instruments exposed to space. The two limit curves in Fig. 17 represent the distance dependence of the radiated magnetic field noise. As is well established, the distance dependence of the magnetic field noise level is related to the size of the current loop of the noise source, and the limit levels are defined in accordance with the case that the level is inversely proportional to the square or the cube of the distance. 


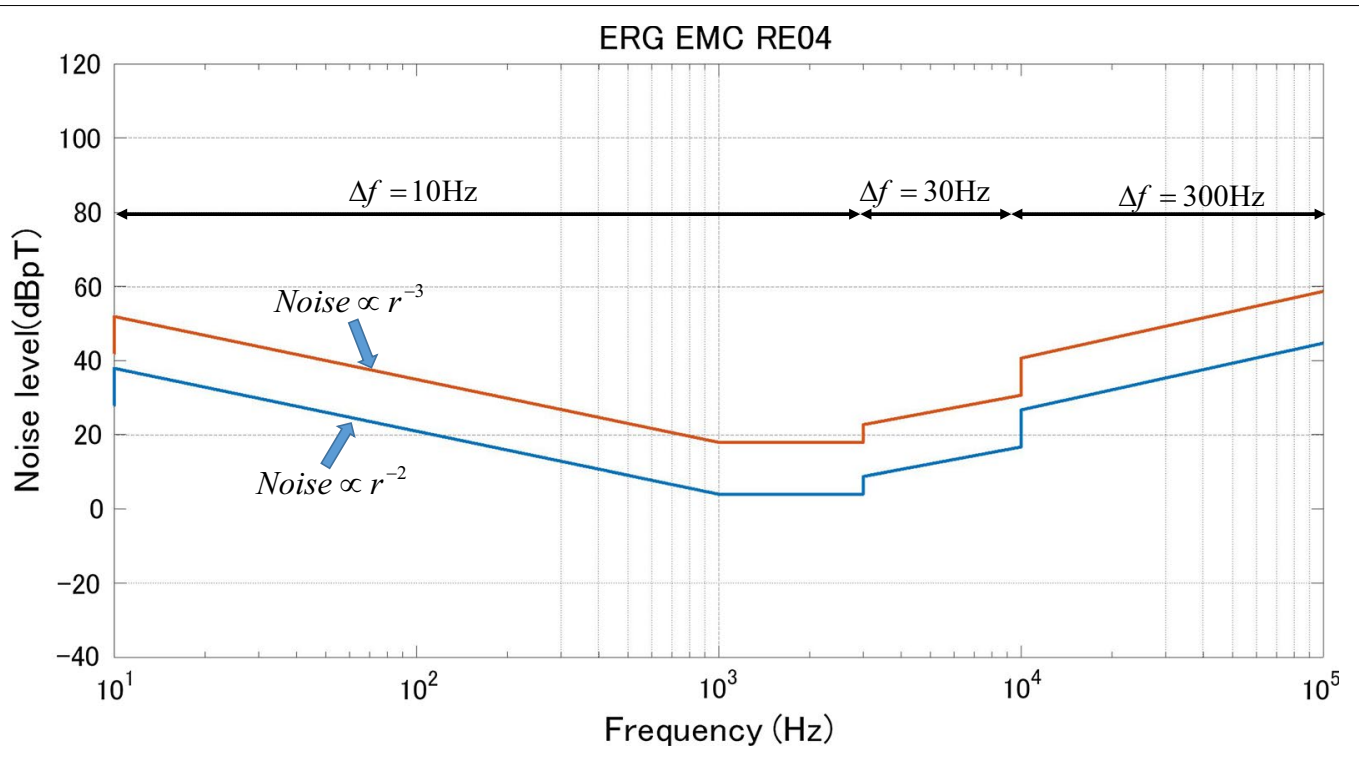

Fig. 17 Limit level for the electromagnetic compatibility (EMC) requirements of the radiated magnetic field noise (RE04)

The Arase missions apply these EMC requirements to all onboard instruments and subsystems. The noise level measurements from each instrument or subsystem were conducted in the electromagnetic shield chamber in JAXA.

\section{Observation strategy}

\section{Features of the PWE functions}

This section describes the observation strategy taking advantage of the specifications of the PWE. The most unique point of the Arase satellite is the installation of the MDR with a capacity of $32 \mathrm{~GB}$. We can store the waveform data in the MDR and transmit them to the ground after data selection, which enables us to obtain scientifically important and/or interesting data efficiently. The maximum length of the continuous waveforms by the WFC is $200 \mathrm{~s}$, and we can store much longer waveforms (a few tens of minutes) continuously when we perform the S-WPIA operation. The detailed data selection process is described later in "Onboard data processing and data reduction" section.

Fine time resolution of the OFA is another feature of the PWE. We nominally operate the OFA with a time resolution of $1 \mathrm{~s}$. This time resolution is sufficient to distinguish the chorus emission from the other plasma waves, even though the time variation of OFA spectra data does not correspond to each chorus element. This feature is also very important in the data selection process described in "Onboard data processing and data reduction" section.

Wide frequency coverage is also an appealing point of the PWE. As the Arase surveys not only in the radiation belt but also inside the plasmasphere changing its altitude between 460 and $\sim 32,000 \mathrm{~km}$, the local cyclotron and plasma frequencies drastically change. The upper frequency bound of the HFA at $10 \mathrm{MHz}$, however, allows for the ability to derive the local electron density from UHR frequency even deep inside the plasmasphere.

Further unique points of the PWE are indebted to the abundance and flexibility of the observation modes and calibration functions utilizing the onboard software combining with the hardware specifications. Detailed specifications and performance of the onboard software are presented in Matsuda et al. (2018).

\section{Onboard data processing and data reduction}

As described in "PWE instrumentation" section, the electric and magnetic data measured by the WPT and MSC are fed into the EWO and HFA, processed by two CPU boards (CPU\#8 and CPU\#9) after analog-to-digital conversion, and sent to the satellite bus system as telemetry data. Table 3 shows the amount of raw data that are digitized by each receiver in the PWE and subsequently stored in the SDRAM on the CPU boards for onboard data processing. As described in "EFD" section, two types of digital data, DPB and SPB, are generated in the EFD. The output from the DPB consists of two component of signals (i.e., $E_{\mathrm{u}}$ and $E_{\mathrm{v}}$ ) (see Fig. 3) with a sampling frequency of $512 \mathrm{~Hz}$ (except for the sweep mode case, in which signals are sampled at $1024 \mathrm{~Hz}$ ). On the other hand, the output from the SPB consists of four components of signals (i.e., $V_{\mathrm{u} 1}, V_{\mathrm{u} 2}, V_{\mathrm{v} 1}, V_{\mathrm{v} 2}$ ) (see Fig. 3) with a sampling frequency of $128 \mathrm{~Hz}$ (Kasaba et al. 2017). As described in "Waveform capture/onboard frequency 
Table 3 Raw data produced in the PWE

\begin{tabular}{|c|c|c|}
\hline Receiver & Data & Total (bps) \\
\hline \multicolumn{3}{|l|}{ EWO-EFD } \\
\hline $\begin{array}{l}\text { DPB } \\
\text { [Sweep] }\end{array}$ & $\begin{array}{l}512 \mathrm{~Hz} \times 16 \mathrm{~b} \text { it } \times 2 \mathrm{ch}[1024 \mathrm{~Hz} \\
\times 16 \mathrm{bit} \times 2 \mathrm{ch} \times 0.5 / 4 \mathrm{~s}]\end{array}$ & $16.4 \mathrm{k}$ \\
\hline SPB & $128 \mathrm{~Hz} \times 16 \mathrm{bit} \times 4 \mathrm{ch}$ & $8.2 \mathrm{k}$ \\
\hline \multicolumn{3}{|c|}{ EWO-WFC/OFA(E) } \\
\hline $\begin{array}{l}\text { Nominal } \\
\text { [for SWPIA] }\end{array}$ & $\begin{array}{l}65.536 \mathrm{kHz} \times 14(16) \mathrm{b} \text { it } \times 2 \mathrm{ch} \\
{[262.144 \mathrm{kHz} \times 14(16) \mathrm{bit} \times 2 \mathrm{c}} \\
\mathrm{h} \times 0.25]\end{array}$ & $2097.1 \mathrm{k}$ \\
\hline \multicolumn{3}{|c|}{ EWO-WFC/OFA(B) } \\
\hline $\begin{array}{l}\text { Nominal } \\
{[\text { LF mode }]}\end{array}$ & $\begin{array}{l}65.536 \mathrm{~Hz} \times 14(16) \mathrm{b} \text { it } \times 3 \mathrm{ch} \\
{[16.384 \mathrm{~Hz} \times 14(16) \mathrm{bit} \times 3 \mathrm{ch}]}\end{array}$ & $3145.7 \mathrm{k}$ or $[786.4 \mathrm{k}]$ \\
\hline \multicolumn{3}{|l|}{ HFA } \\
\hline Spectrum & 32 bit $\times 4$ ch $\times 1024$ pts $/ 0.5 \mathrm{~s}$ & $262 k$ \\
\hline Waveform & 16 bit $\times 2 \mathrm{ch} \times 4096 \mathrm{pts} / 0.5 \mathrm{~s}$ & $262 k$ \\
\hline
\end{tabular}

analyzer" section, the measured data provided to the OFA and WFC are common in the analog-to-digital conversion process within the EWO boards. The OFA data are produced by a frequency analysis in the CPU boards, while the intermittent WFC data are output following digital signal processing: waveform compression for chorus mode or down-sampling for EMIC mode (Matsuda et al. 2018). The raw data for OFA and WFC, therefore, consist of two electric field components and three magnetic field components that are nominally sampled at $65,536 \mathrm{~Hz}$. The WFC/OFA(E) also has a special mode, in accordance with the S-WPIA measurements, in which electric waveforms are sampled at $262,144 \mathrm{~Hz}$ with a duty ratio of $1 / 4$. On the other hand, WFC/OFA (B) has a special operation mode in which waveform acquisition is performed at a $16,384 \mathrm{~Hz}$ sampling rate.

Table 4 shows a list of PWE mission telemetry data that will be produced after the onboard data processing in the CPU boards. In addition to these mission data, the

\section{Table 4 Products contained in the PWE mission data}

\begin{tabular}{|c|c|c|}
\hline Receivers & Telemetry data & \\
\hline \multirow[t]{2}{*}{$\begin{array}{l}\text { EFD } \\
\text { E-field }\end{array}$} & Continuous data & $\begin{array}{l}\text { Spectrum }(\sim 224 \mathrm{~Hz}) \\
\text { Potential }\left(f_{\mathrm{s}}=8 \mathrm{~Hz}\right) \\
\text { Waveform }\left(f_{\mathrm{s}}=64 \mathrm{~Hz} \text { or } 256 \mathrm{~Hz}\right)\end{array}$ \\
\hline & Burst data & Raw Waveform $\left(f_{\mathrm{s}}=512 \mathrm{~Hz}\right)$ \\
\hline $\begin{array}{l}\text { WFC } \\
\text { E\&B fields }\end{array}$ & Burst data & $\begin{array}{l}\text { Waveform } \\
\text { Chorus mode } \\
\text { EMIC mode }\end{array}$ \\
\hline $\begin{array}{l}\text { OFA } \\
\text { E\&B fields }\end{array}$ & Continuous data & $\begin{array}{l}\text { Spectrum (OFA-SPEC) } \\
\text { Spectral matrix (OFA-MATRIX) } \\
\text { Complex spectrum (OFA-COMPLEX) }\end{array}$ \\
\hline $\begin{array}{l}\text { HFA } \\
E(+B) \text { fields }\end{array}$ & Continuous data & $\begin{array}{l}\text { Spectrum }(E \times 2 \text { or } E+B) \\
\text { E-field: } 10 \mathrm{kHz}<f<10 \mathrm{MHz} \\
\text { B-field: } 10 \mathrm{kHz}<f<100 \mathrm{kHz}\end{array}$ \\
\hline
\end{tabular}

housekeeping (HK) data from the PWE are transferred from two CPU boards to the Arase satellite bus system. The mission data produced from the PWE are classified into two types: continuous data and burst data. The data flow produced from the PWE CPU boards is shown in Fig. 18. The continuous data are mission data that are continuously generated during PWE observation operation. The HK data and continuous data are sent directly to the system data recorder (SDR) through the mission data processor (MDP). Since accumulated data in the SDR are all transmitted to ground tracking stations, the continuous data are used for surveying the entire observation. On the other hand, burst data are mission data that are used for detailed measurements, and thus we assign waveform data to this data type. Due to the telemetry capacity limitation, however, all data produced for the burst data cannot be transmitted to the ground. To efficiently obtain scientifically important and/or interesting data, the data produced for the burst data are once stored in the MDR and transmitted to the ground after the data selection process.

We prepare two types of burst modes; waveforms sampled at $65 \mathrm{kHz}$ will be produced as "chorus burst" mode data and waveforms down-sampled at $1024 \mathrm{~Hz}$ will be produced as "EMIC burst" mode data. We also have an "EFD burst" mode, in which the raw data from the EFD are sent to the MDR. The "chorus burst" and the "EMIC burst" are operated exclusively due to the CPU resource limitation, while the "EFD burst" is nominally performed simultaneously to either of these operations. We also provide raw waveform data to the S-WPIA, which is expected to derive direct correlations between wave and particles. During active S-WPIA observation, the highest priority on the Arase mission, the PWE will send raw EWO waveform data to the MDR without any preprocessing in the PWE CPU boards. The PWE burst and the S-WPIA observation are exclusively operated. Because the S-WPIA observation is the highest priority on the Arase mission, the burst observation is suspended when the S-WPIA operation starts and is resumed after the S-WPIA operation is stopped.

The capacity of the MDR is $32 \mathrm{~GB}$, of which $13.2 \mathrm{~GB}$ is allocated to the PWE burst mode and the rest is allocated to the S-WPIA. There are several partitions for the S-WPIA data storage to record several operations of the S-WPIA (Hikishima et al. 2018). As for the PWE burst mode data, we first divide the allocated 13.2 GB into two categories (1) and (2) for each burst mode data, which include EFD, chorus, and EMIC bursts, as shown in Fig. 18. Detailed specifications and contents of the MDR allocated for the PWE burst data are presented in Matsuda et al. (2018). We use one of category as "write category" in which burst data sent from the PWE to the 


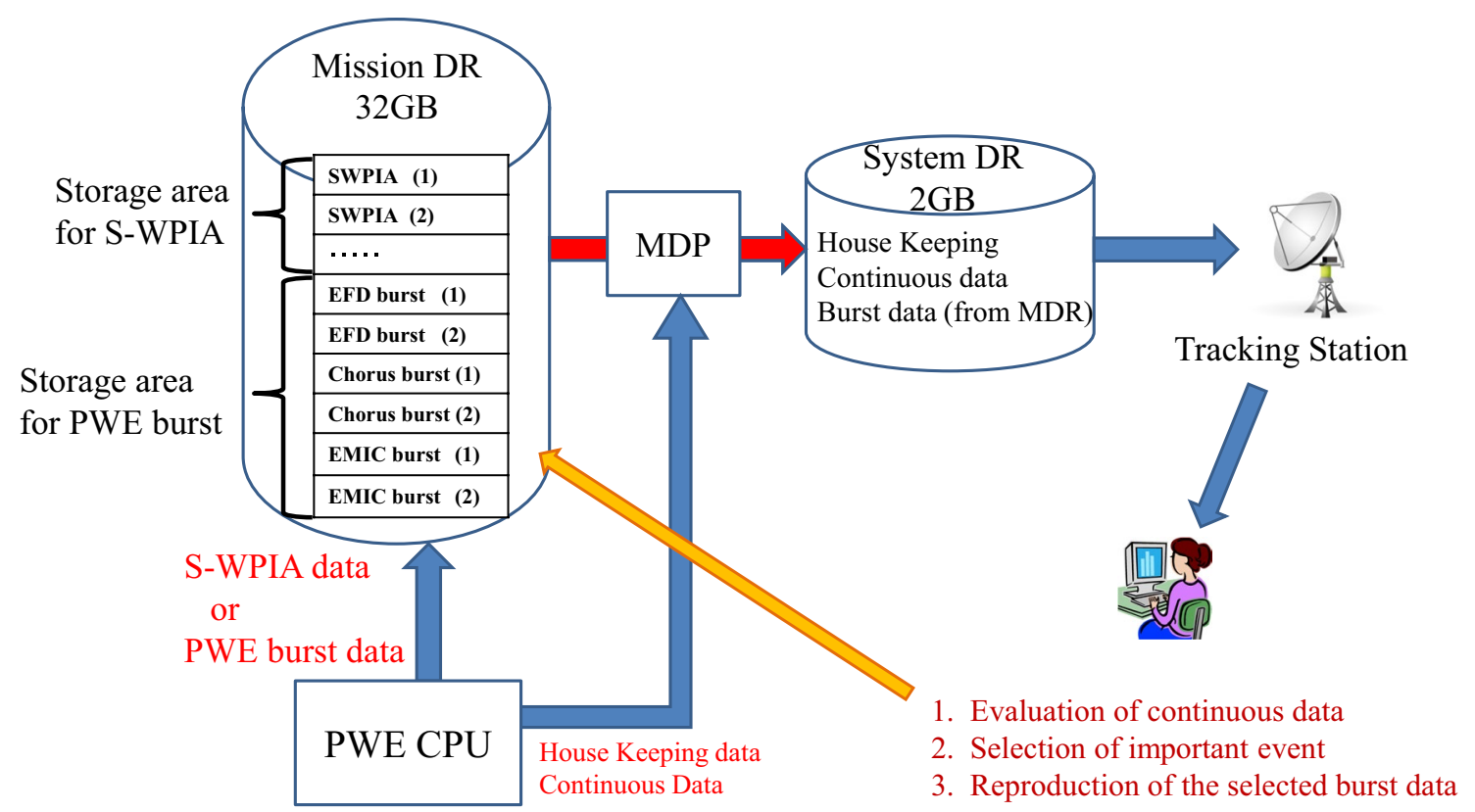

Fig. 18 Data flow of the PWE housekeeping and mission data. The housekeeping and continuous data are sent directly to the system data recorder (SDR) through the mission data processor (MDP). The PWE burst and S-WPIA data are sent to the mission data recorder (MDR) and are stored in a partition corresponding to each data type. In the data selection process, (1) we evaluate the continuous data, (2) select the burst data stored in the MDR, (3) and reproduce the selected burst data. The selected burst data are dumped to the SDR and transmitted to the ground

MDR are written, while we use another category as "read category" from which we reproduce the burst data stored during past observations and send them to the ground. Thanks to the large MDR capacity, we are able to store the burst data in the MDR for several days (nominally 1 week). To select scientifically important and/or interesting events efficiently from the burst data, data selection is performed according to the following processes. (See numbered actions shown in Fig. 18.) As the continuous data are transmitted to the ground within 2 days after observation, (1) we hold an internet meeting once or twice a week to evaluate the continuous data, and (2) we select the burst data stored in the "read category" considering the available telemetry capacity assigned for the burst data. (3) Finally, we upload a timeline command plan to reproduce the selected burst data. The selected burst data are then read by the MDP and are transmitted to the ground via the SDR. In the nominal operation, we exchange the write category and the read category every week.

Table $5 \mathrm{a}$, b shows lists of PWE data produced as continuous data and burst data, respectively, with observation modes and their specifications. For details of product contents, please refer to the following papers: EFD in Kasaba et al. (2017), WFC and OFA in Matsuda et al. (2018) and HFA in Kumamoto et al. (2018).

\section{Nominal observation mode}

Operation of the PWE is performed according to the timeline command plan. The timeline commands are planned and transmitted to the satellite approximately twice a week. During regular operation, we organize timeline commands for the PWE according to the following:

\section{Apogee mode}

When the Arase satellite is located outside of the plasmapause $(L>4)$, the EFD covers the frequency range of ion mode waves such as EMIC, while the WFC/OFA covers the frequency range of VLF waves, such as whistler-mode chorus and plasmaspheric hiss. In particular, when the apogee is located in the dawn side or night side, which corresponds to the first half-year after the launch of Arase, we nominally operate the chorus burst mode in the equatorial region around the apogee to capture chorus waveforms as much as possible. The EFD is operated in "apogee mode," in which we produce the EFD-DPB waveform, sampled at $64 \mathrm{~Hz}$, in cooperation with the MGF to focus on EMIC waves less than $30 \mathrm{~Hz}$.

\section{Perigee mode}


Table 5 Observation modes with their specifications

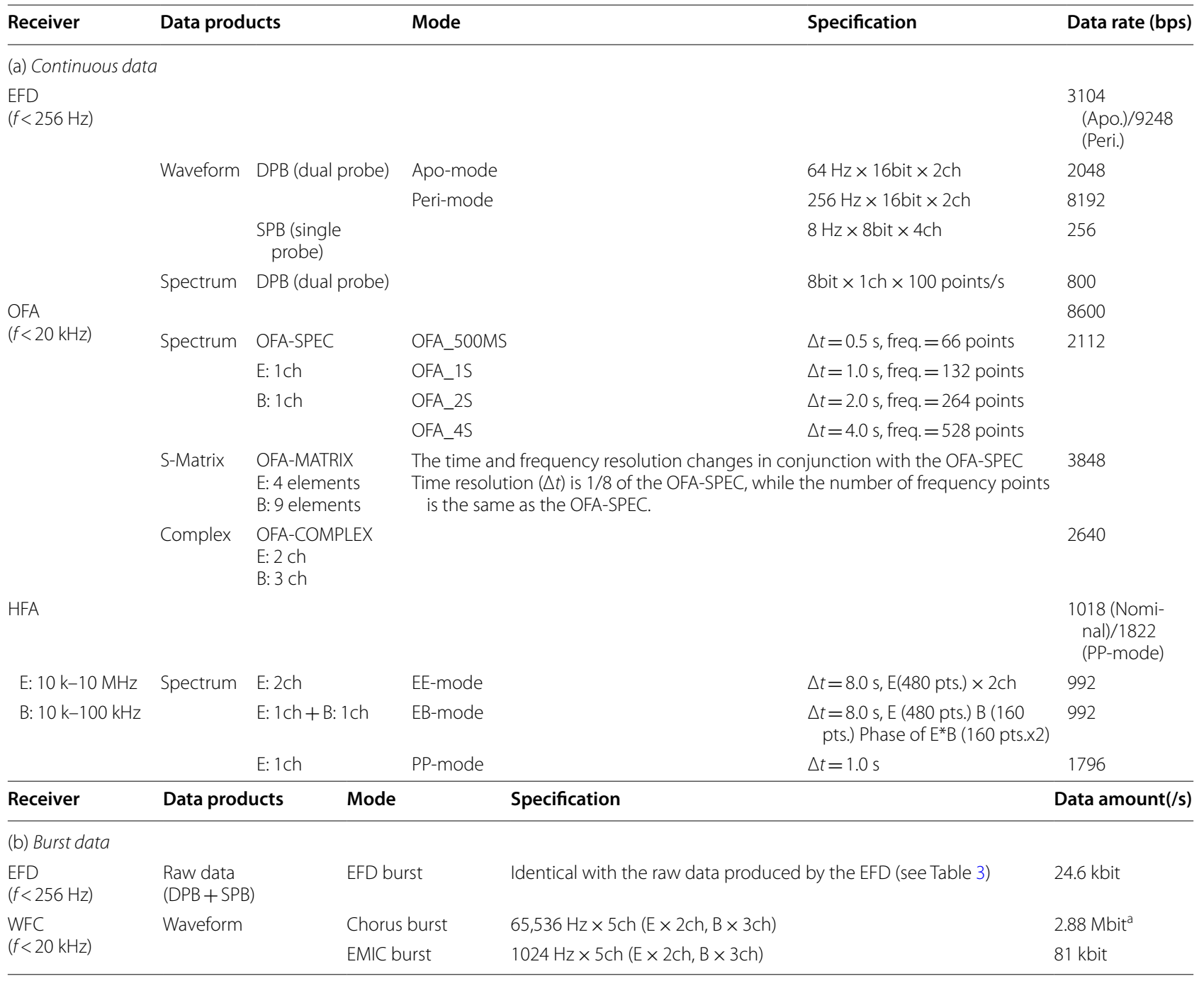

a Data amount in chorus burst mode is compressed to $~ 55 \%$ from the original data

On the other hand, when the Arase is located in the plasmasphere $(L<4)$ where ion cyclotron frequency exceeds $30 \mathrm{~Hz}$, we change the sampling frequency of the EFDDPB to $256 \mathrm{~Hz}$ to cover ion mode waves. During "perigee mode" operation, the MGF will also measure magnetic waveforms sampled at $256 \mathrm{~Hz}$. We operate the EMIC burst mode to measure EMIC waves as well as MSW that are expected at higher frequency ranges (from a few tens $\mathrm{Hz}$ to a few hundred $\mathrm{Hz}$ ).

\section{Plasmapause mode}

To observe spatial variation of electron density at the boundary of the plasmasphere (plasmapause), we operate the plasmapause mode (PP-mode), in which the HFA measures detailed spectrum with a time resolution of $1 \mathrm{~s}$ with an upper frequency limit at $400 \mathrm{kHz}$ (Kumamoto et al. 2018).

\section{Special operations}

In addition to the nominal observation modes introduced in "Nominal observation mode" section, special observation modes and/or specific observation parameters are selected and operated in accordance with campaign observations. Furthermore, as the satellite potential and the electric field antenna impedance change drastically depending on the background plasma density and temperature, we operate a bias sweep operation for the EFD at least once a month. We also implement a software calibration (SW-CAL) for the evaluation of WPT antenna impedance before and after PWE burst operation and/or S-WPIA observation. 


\section{Ground data analysis}

The downloaded telemetry data from the Arase satellite will be stored in the archive system at ISAS/JAXA. We collect all raw data related to the PWE (hereinafter referred to as Level-0 data). Next we divide the Level-0 data into data product species, such as EFD, OFA-E, OFA-B, and HFA (hereinafter referred to as Level-1 data), and record them in Common Data Format (CDF), which is a standardized data format that is suitable for solar-terrestrial physics. As the Level-1 data are still noncalibrated, we calibrate and convert them to Level-2 data in CDF. All of the products shown in Table 4 will be converted into Level-2 CDF data and finally made available to the public at the ERG science center at Nagoya University (Miyoshi et al. 2017).

\section{Initial results}

The Arase has passed its critical operation phase, and we confirmed successful extension of the WPT, MASTMGF, and MAST-SC by January 23, 2017. The initial checkout of the PWE was also conducted and we began to fully operate the PWE in the middle of March 2017. In this section, we introduce initial data from the PWE.

Figure 19 is an example 8-h stack plot of the PWE. The data observed from 05:00 to 13:00 UT on April 11, with corresponding observation statuses, are shown in the upper panels, and the $L$-value, magnetic local time (MLT), magnetic latitude (MLAT), and universal time (UT) are presented at the bottom of the horizontal axis. During this period, the Arase satellite entered into the plasmasphere and passed its perigee at approximately 07:08 UT at an altitude of $425 \mathrm{~km}$ and $~ 15.3$ MLT. Then, the Arase satellite left the plasmasphere and passed its apogee at approximately 11:52 UT at an altitude of $32,300 \mathrm{~km}$ and $~ 3.1$ MLT.

Figure 19a shows a wave spectrogram for $E_{\mathrm{v}}$ electric field component, measured by the HFA, that covered the frequencies from $2 \mathrm{kHz}$ to $10 \mathrm{MHz}$ (vertical axis). Note that the color scales for the HFA are provided in relative values at the right.

Figure 19b, c shows wave spectrograms for electric and magnetic fields observed by the OFA-E and OFA-B, respectively, at frequencies of $32 \mathrm{~Hz}$ to $20 \mathrm{kHz}$. Note that the electric field intensity in Fig. 19b is calculated under the assumptions of theoretical antenna capacitance $(70 \mathrm{pF})$ in a vacuum, typical plasma impedance $(10 \mathrm{M} \Omega)$ and an effective WPT-S length of $15 \mathrm{~m}$. The solid and dotted lines shown in these three spectrograms indicate the local electron cyclotron frequency $\left(f_{c}\right)$ and half of $f_{c}$, respectively, derived from the DC magnetic field measured by the MGF along the trajectory. Figure 19d shows a wave spectrogram for electric fields measured by the EFD, covering the frequencies from 3 to $224 \mathrm{~Hz}$.
Figure 19e, $\mathrm{f}$ shows the absolute value of the $\mathrm{DC}$ electric field and satellite potential measured by the EFD, respectively. The shadows superposed on Fig. 19e, f represent the eclipse period that occurred from 07:53 to 08:34 UT along the trajectory of the Arase. The upper two panels in Fig. 19g represent the times when the PWE burst mode was active, while " $65 \mathrm{k}$ " and " $1 \mathrm{k}$ " shown in each panel correspond to "chorus burst" and "EMIC burst," respectively. The lowest panel in Fig. 19g, with the "WP" abbreviation, shows the time duration of the S-WPIA mode operation. Note that the wideband noises with durations of a few minutes, shown in Fig. 19a, b, at approximately $11: 19$ and 11:35 UT are artificial signals caused by the PWE calibration. The effect of the calibration is also faintly evident in Fig. 19d.

We operated the HFA in "plasmapause mode" from 05:11 to 06:27 UT and from 07:54 to 09:32 UT to measure the detailed electron density profile along the trajectory around the plasmapause, as the Arase was predicted to traverse the $L$-shell region of $\sim 4$. In "plasmapause mode," the upper frequency of the HFA is limited to $400 \mathrm{kHz}$ (Fig. 19a), but the time resolution is $1 \mathrm{~s}$. In the spectrogram obtained by the HFA (Fig. 19a), we can clearly recognize an upper hybrid wave, which was continuously observed at $\sim 100 \mathrm{kHz}$ at 05:00 UT and increased to $\sim 7 \mathrm{MHz}$ at perigee around 07:08 UT. The upper hybrid wave frequency gradually decreased after passing the perigee, but a sudden decrease was observed at 08:57 UT, which indicated the Arase satellite experienced a sudden density gap at plasmapause. It is interesting that at this time, intense electromagnetic waves below $150 \mathrm{~Hz}$ suddenly disappeared, although the wave intensity gradually increased between 08:20 and 08:57 UT as shown in Fig. 19b-d.

From the HFA data, we recognize auroral kilometric radiation (AKR) from 07:30 to 12:00 UT between 100 and $400 \mathrm{kHz}$, although the upper part of the AKR $(f>400 \mathrm{kHz})$ was not measured due to "plasmapause mode" operation. Furthermore, strong electron cyclotron harmonic $(\mathrm{ECH})$ waves were observed after the plasmapause crossing at 08:55 UT and continued until 13:00 UT. The ECHs were also clearly observed by OFA-E (Fig. 19b).

In the OFA data (Fig. 19b, c), hiss-like emissions were observed in the plasmasphere from 05:00 to 06:45 UT and 07:30 to 08:10 UT from several tens of $\mathrm{Hz}$ to $\sim 4 \mathrm{kHz}$. We also observed a frequency gap in the spectra, in which frequencies increased from $\sim 300 \mathrm{~Hz}$ to a few $\mathrm{kHz}$ (05:30-06:50 UT), as the satellite approached to the perigee. A similar gap was observed between 07:20 and 08:15 UT, when frequencies decreased from $10 \mathrm{kHz}$ to $\sim 500 \mathrm{~Hz}$. These gaps seem to correspond with the in situ lower hybrid frequency. After 09:00 UT, when 


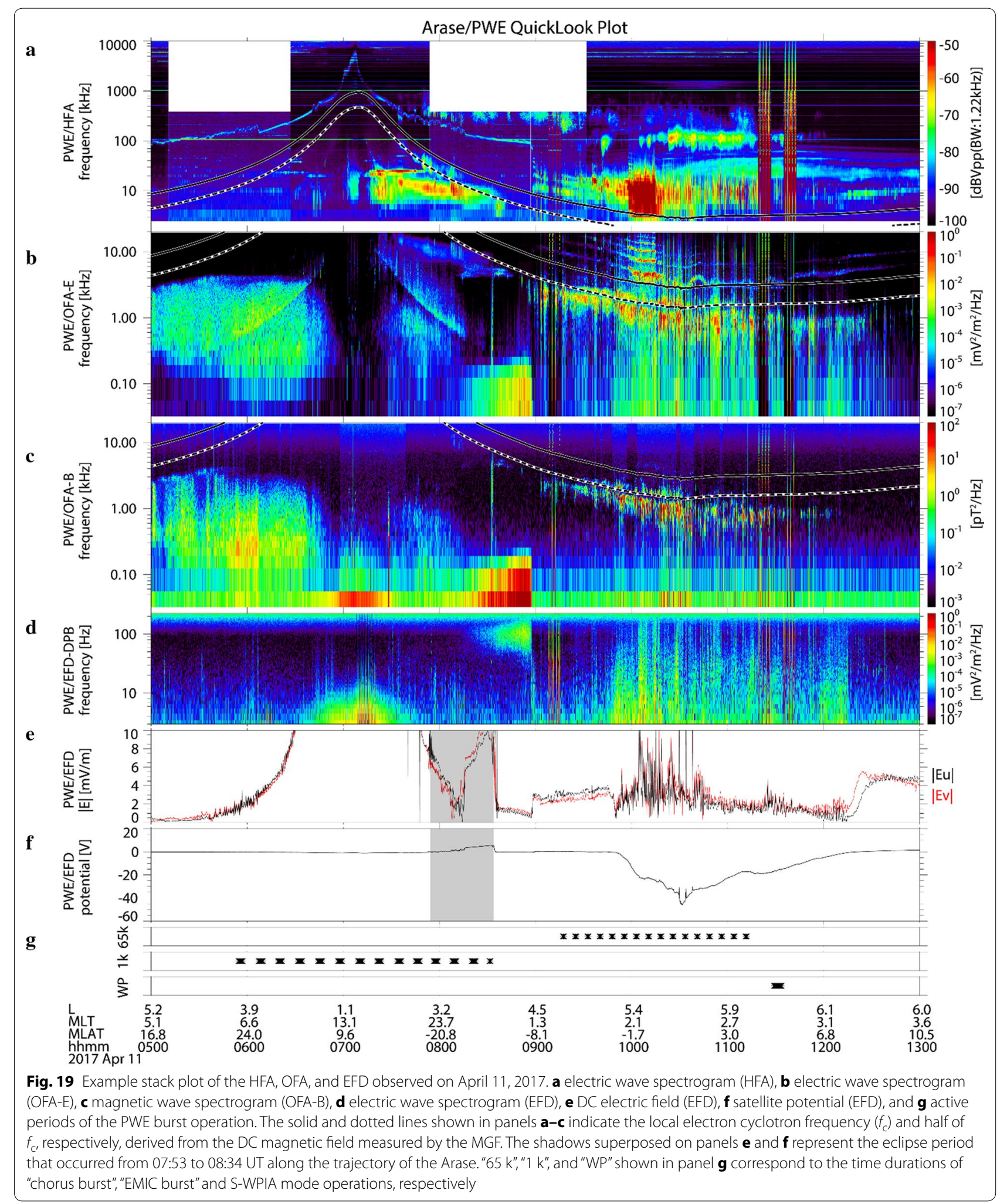


the Arase was orbiting outside of the plasmasphere in the dawn-side region, a dual-band chorus with a gap at approximately $f_{c} / 2$ was observed. We also recognize DC electric field fluctuations and negative satellite charging from 09:50 to 12:10 UT (shown in Fig. 19e, f), which may be related to hot and sub-relativistic electron injections observed by the Arase particle instruments during this period.

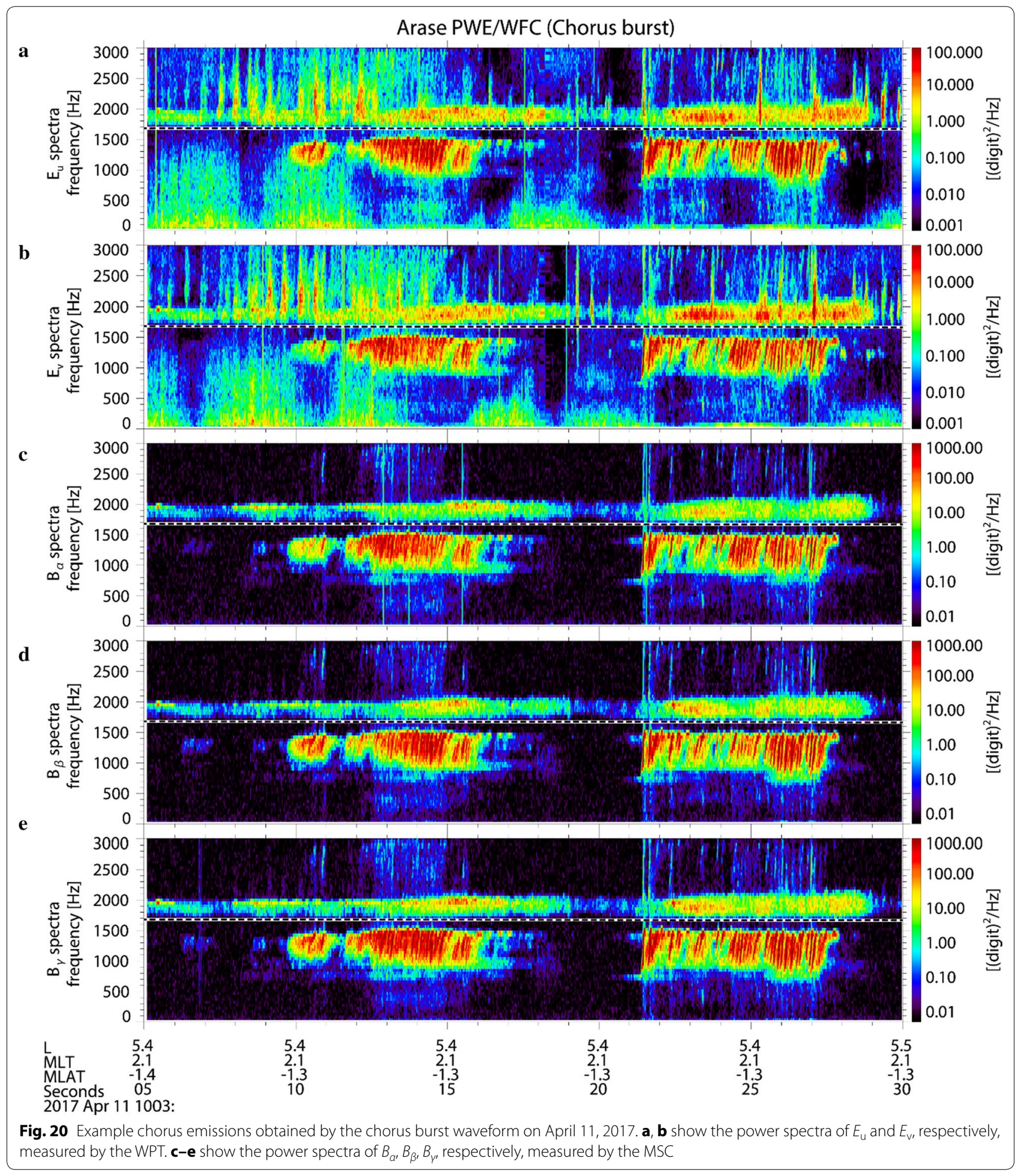


Figure 20 shows example spectra produced from the WFC burst data in "chorus mode" captured between 10:03:05 and 10:03:30 UT on April 11, 2017. We applied a short-time Fourier transform (STFT) analysis every 4096 sampling points (62.5 ms) with a Hanning window that shifted by 256 samples (94\% overlap). Figure 20a, b shows the power spectra of $E_{\mathrm{u}}$ and $E_{\mathrm{v}}$ (two channels of waveforms in the electric field components), respectively, measured by the WPT. Figure 20c-e shows the power spectra of $B_{\alpha}, B_{\beta}, B_{\gamma}$ (three channels of waveforms in the magnetic field components), respectively, measured by the MSC. The dotted lines shown in these spectrograms indicate half of the local $f_{\mathrm{c}}$, derived from DC magnetic field measured by the MGF. During this period, the upper and lower band chorus waves were simultaneously observed with a clear frequency gap at $f_{\mathrm{c}} / 2$. Each chorus element shows a frequency increase that is characteristic of a "rising-tone" chorus.

\section{Conclusion}

In this paper, we introduced the specifications and initial results from the PWE on board the Arase satellite that was launched on December 20, 2017. Thus far, the PWE has been successfully operated and has acquired highquality continuous and burst data.

Table 6 summarizes the burst mode data that have been obtained. The amounts in the table represent total amounts of data that are selected from the MDR and downloaded to the ground between March 21, 2017, and August 4, 2017. Note that the S-WPIA mode data in the table include only the PWE waveform data. The time intervals in parentheses represent the durations of the observation data that were downloaded to the ground and were produced by the PWE, respectively. As shown in Table 6, waveform data exceeding 50 GB were acquired. We expect that analyses of these data will clarify the micro-physics of wave-particle interaction in the Earth's inner magnetosphere.

Currently, we are conducting detailed calibration of the PWE data, according to the regular operation of the onboard calibration functions. While the magnetic field calibration is almost complete, the electric

\section{Table 6 Total amount of waveform data obtained by PWE burst and S-WPIA operations from March 21, 2017 to August 4, 2017}

\begin{tabular}{ll}
\hline Observation mode & $\begin{array}{l}\text { Amounts of data (received/ } \\
\text { measured) }\end{array}$ \\
\hline Chorus burst mode & $32.5 \mathrm{~GB}(1644 \mathrm{~min} / 3884 \mathrm{~min})$ \\
EMIC burst mode & $5.26 \mathrm{~GB}(16,420 \mathrm{~min} / 28,420 \mathrm{~min})$ \\
S-WPIA mode (waveform data only) & $12.8 \mathrm{~GB}(577 \mathrm{~min} / 1135 \mathrm{~min})$ \\
\hline
\end{tabular}

field calibration is ongoing, as it is essential to evaluate the variation of the WPT antenna impedance due to plasma density and temperature influences. Furthermore, in waveform data calibration, it is necessary to calibrate considering not only gain but also phase of the WFC/OFA frequency responses, as the waveform shape depends on both. Following these calibration processes, the data are scheduled to be released as Level-2 data from the ERG Science Center in Nagoya University.

Simultaneous observations with other satellites and ground observation networks will be important in future studies. In particular, cooperative observations with the Van Allen Probes, THEMIS (Angelopoulos 2008) and MMS (Burch et al. 2016) will allow comprehensive understanding of plasma wave generation and propagation characteristics in the inner magnetosphere. Conjugate observations of the Arase satellite and the ground observation network are also important in clarifying particle precipitation processes, as consequences of waveparticle interactions. When the nominal operation of the Arase began in the mid-March, 2017, campaign observations were conducted with ground observation networks, such as EISCAT and the PWING project (Shiokawa et al. 2017). We expect comparisons of the simultaneous observation data from these ground-network stations with Arase data will produce many new scientific achievements.

\section{Abbreviations}

AGC: automatic gain control; AKR: auroral kilometric radiation; ASIC: application-specific integrated circuits; CDF: common data format; CE: conducted emission; DPB: double probe; EFD: electric field detector; EMC: electromagnetic compatibility; EMIC: electromagnetic ion cyclotron wave; EWO: EFD/ WFC/OFA; FPGA: field-programmable gate array; HEP: high-energy electron experiments; HFA: high-frequency analyzer; HK: housekeeping; HPF: high-pass Filter; HSS: high-speed solar wind streams; IPD: instruments power distributer; LEP: low-energy particle experiments; LPF: low-pass Filter; MAST: extendable mast; MDR: mission data recorder; MDP: mission data processor; MEP: medium-energy particle experiments; MGF: magnetic field experiment; MMO: mercury magnetospheric orbiter; MLAT: magnetic latitude; MLT: magnetic local time; MSC: magnetic search coil; MSPS: mega-samples per second; MSW: magnetosonic wave; MWE: MAST/WPT-S electronics; OFA: onboard frequency analyzer; PSU: power supply unit; PWE: Plasma Wave Experiment; PWI: Plasma Wave Investigation; RBSP: radiation belt storm probes; RE: radiated emission; SDR: system data recorder; SDRAM: synchronous dynamic random access memory; SGA: spinning satellite geometry axis coordinate; SPB: single probe; SW-CAL: software calibration; S-WPIA: software-type wave-particle interaction analyzer; UHR: upper hybrid resonance; UT: universal time; WFC: waveform capture; WPT: wire probe antenna; XEP: extremely high-energy electron experiments.

\section{Authors' contributions}

YK is the PI of the PWE and the lead author of this article. YK is the Co-PI of the PWE responsible for the development of the WPT and EFD. HK is the Co-PI and the engineering manager of the PWE and the PI of the S-WPIA. SY is the Co-PI of the PWE responsible for the development of the MSC. KI is a Co-I of the PWE responsible for the development of the EFD. AK and FT are Co-Is of the PWE responsible for the development of the HFA. MO is the Co-l of the PWE responsible for the development of the MSC. SM and TI are Co-Is of the PWE responsible for the development of the onboard software. YM is the 
project scientist of the ERG project. MH is the Co-I of the PWE and S-WPIA responsible for software development related to the interface between PWE and S-WPIA. YK is the Co-l of the PWE and the S-WPIA responsible for the interface between PWE and S-WPIA. MO is the Co-I of the PWE who designed the specifications of the OFA-MATRIX and OFA-COMPLEX. MS is the Co-I of the PWE and contributed to the designing of the data processing interface for the PWE in the ERG science center. AM is the PI of the MGF and responsible for the deployment of the MAST and WPT. IS is the project manager of the ERG mission. All authors read and approved the final manuscript.

\section{Author details}

1 Graduate School of Natural Science and Technology, Kanazawa University, Kakuma, Kanazawa 920-1192, Japan. ${ }^{2}$ Graduate School of Science, Tohoku University, Aoba-ku, Sendai 980-8578, Japan. ${ }^{3}$ Research Institute for Sustainable Humanosphere, Kyoto University, Gokasho, Uji 611-0011, Japan. ${ }^{4}$ Toyama Prefectural University, Toyama, Japan. ${ }^{5}$ Nagoya University, Nagoya, Japan. ${ }^{6}$ ISAS, JAXA, Kanazawa, Japan.

\section{Acknowledgements}

The PWE team deeply acknowledges the great efforts of the late Professor Emeritus T. Ono, the original PI of the ERG project. We also would like to express our sincere thanks to ISAS/JAXA for the support of the PWE development and successful launch of the Arase satellite. The authors wish to express their deep appreciation to Professors I. Nagano, A. Morioka, T. Mukai and T. Okada for their valuable comments and encouragement. We are greatly indebted to Messrs. M. Koyama, T. Miyabara, K. Genba, S. Sasahara, and S. Eguchi (Mitsubishi Heavy Industries Ltd.) for their fabrication, total arrangement of the PWE, and middleware development for the onboard CPU. We also express our thanks to Messrs. O. Nara, T. Watanabe, T. Suzuki, I. Tanaka, and K. Tanimoto (Meisei Electric Co. Ltd.) for their fabrication of the HFA, and to Messrs. O. Maeda, T. Yumoto, T. Sasaki, H. Sato, Y. Hayasaka, and Ms. Y. Ono (NIPPI Co. Ltd.) for their fabrication of the WPT, MSC and MAST. The authors wish to express their thanks to Drs. S. Nakazawa and M. Mita, Mr. Y. Sato, and Ms. M. Murashima for their support during the electromagnetic compatibility tests. We are also grateful to Drs. T. Takashima, K. Asamura, Ms. E. Ogawa, and all colleagues of the Arase project team members. The PWE integration tests were conducted using the PEMSEE system at Research Institute for Sustainable Humanosphere, Kyoto University, Japan.

\section{Competing interests}

The authors declare that they have no competing interests.

\section{Availability of data and materials}

The PWE data will be distributed from the ERG Science Center operated by ISAS/JAXA and ISEE/Nagoya University.

\section{Consent for publication}

Not applicable.

\section{Ethics approval and consent to participants}

Not applicable.

\section{Funding}

This research was partially supported by a Grant-in-Aid for Scientific Research from the Japan Society for the Promotion of Science (\#16H04056 and \#16H01172).

\section{Publisher's Note}

Springer Nature remains neutral with regard to jurisdictional claims in published maps and institutional affiliations.

Received: 6 September 2017 Accepted: 14 April 2018

Published online: 21 May 2018

\section{References}

Angelopoulos V (2008) The THEMIS mission. Space Sci Rev. https://doi. org/10.1007/s11214-008-9336-1
Asamura K et al (2018) Low-energy particle experiments -ion analyzer (LEPi) onboard the ERG (Arase) satellite. Earth Planets Space. https://doi. org/10.1186/s40623-018-0846-0

Baker DN et al (2014) An impenetrable barrier to ultrarelativistic electrons in the Van Allen radiation belts. Nature 515:531-534. https://doi. org/10.1038/nature13956

Breneman AW et al (2015) Global-scale coherence modulation of radiationbelt electron loss from plasmaspheric hiss. Nature 523:193-195. https:// doi.org/10.1038/nature14515

Burch JL et al (2016) Magnetospheric multiscale overview and science objectives. Space Sci Rev. https://doi.org/10.1007/s11214-015-0164-9

Cattell C et al (2008) Discovery of very large amplitude whistler-mode waves in Earth's radiation belts. Geophys Res Lett 35:L01105. https://doi. org/10.1029/2007GL032009

European cooperation for space standardization (ECSS-E-ST-50-12C) (2008) Space engineering, SpaceWire-Links, nodes, routers and networks, European Space Agency

European cooperation for space standardization (ECSS-E-ST-50-51C) (2010) Space engineering, SpaceWire protocol integration, European Space Agency

European cooperation for space standardization (ECSS-E-ST-50-52C) (2010) Space engineering, SpaceWire-Remote memory access protocol, European Space Agency

Fraser BJ, Nguyen TS (2001) Is the plasmapause a preferred source region of electromagnetic ion cyclotron waves in the magnetosphere? J Atmos Sol Terr Phys 63:1225-1247

Fukuhara $\mathrm{H}$ et al (2009) A new instrument for the study of wave-particle interactions in space: one-chip Wave-Particle Interaction Analyzer. Earth Planets Space 61:BF03353183. https://doi.org/10.1186/BF03353183

Fukunishi $\mathrm{H}$ et al (1990) Magnetic field observations on the Akebono (EXOS-D) satellite. J Geomagn Geoelectr 42:385-409

Hikishima M et al (2018) Data processing in the Software-type wave-particle interaction analyzer on board the Arase satellite. Earth Planets Space. https://doi.org/10.1186/s40623-018-0856-y

Horne RB et al (2005) Timescale for radiation belt electron acceleration by whistler mode chorus waves. J Geophys Res 110:A03225. https://doi. org/10.1029/2004JA010811

Horne RB et al (2007) Electron acceleration in the Van Allen radiation belts by fast magnetosonic waves. Geophys Res Lett 34:L17107. https://doi. org/10.1029/2007GL030267

Jordanova VK et al (2008) Relativistic electron precipitation by EMIC waves from self-consistent global simulations. J Geophys Res 113:A00A10. https://doi.org/10.1029/2008JA013239

Kasaba Y et al (2010) The plasma wave investigation (PWI) onboard the BepiColombo/MMO: first measurement of electric fields, electromagnetic waves, and radio waves around Mercury. Planet Space Sci 58:238-278

Kasaba Y et al (2017) Wire Probe Antenna (WPT) and Electric Field Detector (EFD) of Plasma Wave Experiment (PWE) aboard ARASE: specifications and Initial Evaluation results. Earth Planets Space. https://doi.org/10.1186/ s40623-017-0760-X

Kasahara Y et al (1992) Ion cyclotron emissions observed by the satellite Akebono in the vicinity of the magnetic equator. Radio Sci 27:347-362

Kasahara Y et al (1994) Propagation characteristics of the ELF emissions observed by the satellite Akebono in the magnetic equatorial region. Radio Sci 29:751-767

Kasahara Y et al (2004) Whistler mode chorus observed around the plasmapause during magnetic storms. In: Frontiers of magnetospheric physics, COSPAR Colloquia Series. Elsevier, Amsterdam, vol 16, pp 228-234

Kasahara Y et al (2009) Simultaneous satellite observations of VLF chorus, hot and relativistic electrons in a magnetic storm "recovery" phase. Geophys Res Lett 36:L01106. https://doi.org/10.1029/2008GL036454

Katoh Y, Omura Y (2007) Computer simulation of chorus wave generation in the Earth's inner magnetosphere. Geophys Res Lett 34:L03102. https:// doi.org/10.1029/2006GL028594

Katoh Y, Omura Y (2016) Electron hybrid code simulation of whistler-mode chorus generation with real parameters in the Earth's inner magnetosphere. Earth Planets Space 68:192. https://doi.org/10.1186/ s40623-016-0568-0

Katoh Y et al (2013) Significance of wave-particle interaction analyzer for direct measurements of nonlinear wave-particle interactions. Ann Geophys 31:503-512. https://doi.org/10.5194/angeo-31-503-2013 
Katoh Y et al (2018) Software-type wave-particle interaction analyzer on board the ARASE satellite. Earth Planets Space. https://doi.org/10.1186/ s40623-017-0771-7

Kazama et al (2017) Low-energy particle experiments - electron analyzer (LEPe) onboard the Arase spacecraft. Earth Planets Space. https://doi. org/10.1186/s40623-017-0748-6

Kimura et al (1990) VLF observation by the Akebono (EXOS-D) satellite. J Geomagn Geoelectr 42:459-478

Kitahara M, Katoh Y (2016) Method for direct detection of pitch angle scattering of energetic electrons caused by whistler mode chorus emissions. J Geophys Res Space Phys 121:5137-5148. https://doi. org/10.1002/2015JA021902

Kletzing C et al (2013) The electric and magnetic field instrument suite and integrated science and integrated science (EMFISIS) on RBSP. Space Sci Rev 179:127-181. https://doi.org/10.1007/s11214-013-9993-6

Kubota Y et al (2015) Relativistic electron precipitation induced by EMIC-triggered emissions in a dipole magnetosphere. J Geophys Res Space Phys 120:4384-4399. https://doi.org/10.1002/2015JA021017

Kumamoto A et al (2018) High Frequency Analyzer (HFA) of Plasma Wave Experiment (PWE) onboard the Arase spacecraft. Earth Planets Space. https://doi.org/10.1186/s40623-018-0854-0

Kuroda $Y$ et al (2011) Development of processor for both space and commercial use: development of SOI-SoC. Aeronaut Space Sci Jpn 59:149-154. https://doi.org/10.14822/kjsass.59.688_149(in Japanese)

Laakso $\mathrm{H}$ et al (1990) Magnetosonic waves above $f_{\mathrm{c}}\left(\mathrm{H}^{+}\right)$at geostationary orbit: GEOS 2 results. J Geophys Res 95(A7):10609-10621. https://doi. org/10.1029/JA095iA07p10609

Matsuda S et al (2014a) Electromagnetic ion cyclotron waves suggesting minor ion existence in the inner magnetosphere observed by the Akebono satellite. J Geophys Res Space Phys 119:4348-4357. https://doi. org/10.1002/2013JA019370

Matsuda S et al (2014b) High-altitude $M / Q=2$ ion cyclotron whistlers in the inner magnetosphere observed by the Akebono Satellite. Geophys Res Lett 41:3759-3765. https://doi.org/10.1002/2014GL060459

Matsuda S et al (2015) M/Q = 2 ion distribution in the inner magnetosphere estimated from ion cyclotron whistler waves observed by the Akebono satellite. J Geophys Res Space Phys 120:2783-2795. https://doi. org/10.1002/2014JA020972

Matsuda S et al (2016) Variation in crossover frequency of EMIC waves in plasmasphere estimated from ion cyclotron whistler waves observed by Van Allen Probe A. Geophys Res Lett 43:28-34. https://doi. org/10.1002/2015GL066893

Matsuda S et al (2018) Onboard Software of Plasma Wave Experiment aboard Arase: instrument Management and Signal Processing of Waveform Capture/Onboard Frequency Analyzer. Earth Planets Space. https://doi. org/10.1186/s40623-018-0838-0

Matsumoto $\mathrm{H}$ et al (1994) Plasma wave observations with GEOTAIL spacecraft. J Geomagn Geoelectr 46:59-95. https://doi.org/10.5636/jgg.46.59

Matsuoka A et al (2018) The ARASE (ERG) magnetic field investigation. Earth Planets Space. https://doi.org/10.1186/s40623-018-0800-1

Mauk BH et al (2013) Science objectives and rationale for the radiation belt storm probes mission. Space Sci Rev 179:3-27. https://doi.org/10.1007/ s11214-012-9908-y

Meredith NP et al (2008) Survey of magnetosonic waves and proton ring distributions in the Earth's inner magnetosphere. J Geophys Res 113:A06213. https://doi.org/10.1029/2007JA012975

Min K et al (2012) Global distribution of EMIC waves derived from THEMIS observations. J Geophys Res 117:A05219. https://doi. org/10.1029/2012JA017515

Miyoshi Y et al (2012) The Energization and Radiation in Geospace (ERG) project, in dynamics of the earth's radiation belts and inner magnetosphere In: Summers D, Mann IR, Baker DN, Schulz M (eds), pp 103-116. https:// doi.org/10.1029/2012gm001304

Miyoshi et al. (2017) Geospace exploration project: Arase (ERG). In: IOP Conference Series: Journal of Physics: Conference Series, vol 869. https://doi. org/10.1088/1742-6596/869/1/012095

Miyoshi Y et al (2003) Rebuilding process of the outer radiation belt during the 3 November 1993 magnetic storm: NOAA and Exos-D observations. J Geophys Res 108(A1):1004. https://doi.org/10.1029/2001JA007542
Miyoshi Y et al (2008) Precipitation of radiation belt electrons by EMIC waves, observed from ground and space. Geophys Res Lett 35:L23101. https:// doi.org/10.1029/2008GL035727

Miyoshi Y et al (2013) High-speed solar wind with southward interplanetary magnetic field causes relativistic electron flux enhancement of the outer radiation belt via enhanced condition of whistler waves. Geophys Res Lett 40:4520-4525. https://doi.org/10.1002/grl.50916

Nakamura S et al (2015) Subpacket structures in EMIC rising tone emissions observed by the THEMIS probes. J Geophys Res Space Phys 120:73187330. https://doi.org/10.1002/2014JA020764

Nishimura Y et al (2007) Evolution of ring current and radiation belt particles under the influence of storm-time electric fields. J Geophys Res 112:A06241. https://doi.org/10.1029/2006JA012177

Omura Y (2014) Theory and simulations of nonlinear wave-particle interactions in planetary radiation belts. Radio Sci Bull 349:52-58

Omura Y et al (2008) Theory and simulation of the generation of whistler-mode chorus. J Geophys Res 113:A04223. https://doi. org/10.1029/2007JA012622

Omura Y et al (2015) Formation process of relativistic electron flux through interaction with chorus emissions in the Earth's inner magnetosphere. J Geophys Res Space Phys 120:9545-9562. https://doi. org/10.1002/2015JA021563

Ozaki M et al (2014) Current-sensitive CMOS preamplifier for investigating space plasma waves by magnetic search coils. IEEE Sens J 14:421-429

Ozaki M et al (2016) Development of an ASIC preamplifier for electromagnetic sensor probes for monitoring space electromagnetic environments. Earth Planets Space. https://doi.org/10.1186/s40623-016-0470-9

Ozaki M et al (2018) Magnetic Search Coil (MSC) of Plasma Wave Experiment (PWE) aboard the Arase (ERG) satellite. Earth Planets Space. https://doi. org/10.1186/s40623-018-0837-1

Paulikas GA, Blake JB (1979) Effects of the solar wind on magnetospheric dynamics: Energetic electrons at the synchronous orbit, Quantitative Modeling of Magnetospheric Processes, 21. Geophys, Monograph Series

Reeves GD et al (2003) Acceleration and loss of relativistic electrons during geomagnetic storms. Geophys Res Lett. https://doi. org/10.1029/2002GL016513

Sakaguchi K et al (2013) Akebono Observations of EMIC Waves in the Slot Region of the Radiation Belts. Geophys Res Lett. https://doi. org/10.1002/2013GL058258

Santolik O et al (2004) Systematic analysis of equatorial noise below the lower hybrid frequency. Ann Geophys 22:2587-2595. https://doi.org/10.5194/ angeo-22-2587-2004

Santolik O et al (2016) Propagation of equatorial noise to low altitudes: decoupling from the magnetosonic mode. Geophys Res Lett. https://doi. org/10.1002/2016GL069582

Shiokawa et al (2017) Ground-based instruments of the PWING project to investigate dynamics of the inner magnetosphere at subauroral latitudes as a part of the ERG-ground coordinated observation network. Earth Planets Space. https://doi.org/10.1186/s40623-017-0745-9

Shoji M, Omura Y (2014) Spectrum characteristics of electromagnetic ion cyclotron triggered emissions and associated energetic proton dynamics. J Geophys Res Space Phys 119:3480-3489. https://doi. org/10.1002/2013JA019695

Shprits YY et al (2016) Wave-induced loss of ultra-relativistic electrons in the Van Allen radiation belts. Nat Commun. https://doi.org/10.1038/ ncomms 12883

Summers D et al (1998) Relativistic theory of wave-particle resonant diffusion with application to electron acceleration in the magnetosphere. J Geophys Res 103(A9):20487-20500. https://doi.org/10.1029/98JA01740

Takashima T et al (2018) Design of mission network system with SpaceWire for scientific payloads onboard the ARASE spacecraft. Earth Planets Space. https://doi.org/10.1186/s40623-018-0839-z

Thaller SA et al (2015) Van Allen Probes investigation of the large-scale duskward electric field and its role in ring current formation and plasmasphere erosion in the 1 June 2013 storm. J Geophys Res Space Phys 120:4531-4543. https://doi.org/10.1002/2014JA020875

Thorne RM (2010) Radiation belt dynamics: the importance of waveparticle interactions. Geophys Res Lett 37:L22107. https://doi. org/10.1029/2010GL044990 
Turner DL et al (2013) On the storm-time evolution of relativistic electron phase space density in Earth's outer radiation belt. J Geophys Res Space Phys 118:2196-2212. https://doi.org/10.1002/jgra.50151

Usanova ME et al (2012) THEMIS observations of electromagnetic ion cyclotron wave occurrence: dependence on $\mathrm{AE}, \mathrm{SYMH}$, and solar wind dynamic pressure. J Geophys Res 117:A10218. https://doi. org/10.1029/2012JA018049
Wygant JR et al (2013) The electric field and waves instruments on the radiation belt storm probes mission. Space Sci Rev 179:183-220. https://doi. org/10.1007/s11214-013-0013-7

Young DT et al (1981) Wave-particle interactions near $\Omega_{\mathrm{He}}$ observed on GEOS 1 and 21 . Propagation of ion cyclotron waves in $\mathrm{He}^{+}$-rich plasma. J Geophys Res 86(A8):6755-6772. https://doi.org/10.1029/JA086iA08p06755

\section{Submit your manuscript to a SpringerOpen ${ }^{\circ}$ journal and benefit from:}

- Convenient online submission

- Rigorous peer review

- Open access: articles freely available online

- High visibility within the field

- Retaining the copyright to your article

Submit your next manuscript at $\boldsymbol{\nabla}$ springeropen.com 